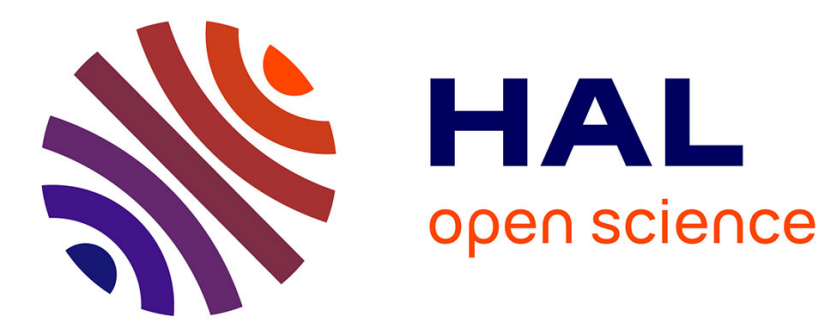

\title{
Inscriptions peintes sur les amphores gauloises
}

Fanette Laubenheimer

\section{To cite this version:}

Fanette Laubenheimer. Inscriptions peintes sur les amphores gauloises. Gallia - Archéologie de la France antique, 2004, L'écriture dans la société gallo-romaine, 61, pp.153-171. 10.3406/galia.2004.3192 . hal-01910882

\section{HAL Id: hal-01910882 \\ https://hal.science/hal-01910882}

Submitted on 20 Jan 2020

HAL is a multi-disciplinary open access archive for the deposit and dissemination of scientific research documents, whether they are published or not. The documents may come from teaching and research institutions in France or abroad, or from public or private research centers.
L'archive ouverte pluridisciplinaire HAL, est destinée au dépôt et à la diffusion de documents scientifiques de niveau recherche, publiés ou non, émanant des établissements d'enseignement et de recherche français ou étrangers, des laboratoires publics ou privés.

\section{(ㅇ)(1) $\$$}

Distributed under a Creative Commons Attribution - NonCommercial - NoDerivatives| 4.0 


\title{
INSCRIPTIONS PEINTES
}

\section{SUR LES AMPHORES GAULOISES}

\author{
Fanette LAUBENHEIMER
}

\begin{abstract}
Mots-clés. Inscriptions peintes, amphores, poisson, olives, vin, nombre.
Résumé. Les inscriptions peintes sur les amphores au moment de leur première utilisation donnent des indications sur leur contenu, sa qualité, sa quantité, voire son âge ou son origine... Elles traduisent, pour le commerce des denrées, le rôle fondamental de ce que l'on écrit et de ce que l'on donne à lire. Le corpus des tituli picti sur amphores gauloises rassemblé ici compte plus de cent cinquante numéros qui permettent de faire le point de la façon dont, en Gaule, on écrivait sur les amphores à poisson, à olives ou à vin en s'inspirant de modèles connus, mais aussi aver une certaine originalité.
\end{abstract}

Key-words. Tituli picti, amphorae, fish, olives, wine, numeral.

Abstract. The tituli picti of amphorae give at the time of the first utilization indications on their content, its quality, the amount, eventually its age or origin. They reflect for the trade of goods, the function of what is written and given to be read. A corpus of more than 150 tituli picti on (arulish amphorae recorded here enables us to study the method of writing in Gaul on fish, wine and olive amphorae, in copying known models with a touch of originality.

Translation : Isabelle FAlDLET

schlagwörter. Pinselaufschriften, Amphoren, Fisch, Oliven, Wein, Anzahl.

Zusammenfassung. Die bei der Erstverwendung auf den Amphoren angebrachten Pinselaufschriften geben Auskunft über den Inhalt, 'essen Qualitäl und Quantität sowie sein Alter und seine Herkunft. Sie verraten die fundameniale Rolle dessen was beim Handel mit Lebensmitteln aufgeschrieben wird und dessen was gelesen werden soll. Das hier zusammengestellte Korpus von tituli picti auf gallischen Amphoren umfaßt mehr als 150 Einzelbelege. Zusammen fassend ergibt sich, daß in Gallien Fisch-, Oliven- und Weinamphoren beschriftet wurden. Dabei orientierte man sich an bekannten Vorbildern, entwickelte aber auch eine gewisse Eigenständigkeit.

Übersetzung: Stefan WIRTH

Écrire sur les amphores est une chose habituelle dans l'Antiquité. Crues, elles sont parfois timbrées avec une matrice portant le plus souvent un nom de personne en relation avec la fabrique (Manacorda, Panella, 1993) et peuvent recevoir aussi une sorte de marque de tâcheron du doigt du potier, ou bien un graffite plus élaboré (Rodriguez-Almeida, 1993). L.orsqu'elles sont cuites et remplies, on peindra sur le col ou sur la panse toutes sortes d'indications, apposées progressivement lors des étapes que suivra la marchandise : il s'agit du produit transporté (qualité, quantité, origine, âge...), du négociant, voire du destinataire, des contrôleurs, etc. Sur les bouchons cachetés, se trouvent aussi des symboles et les noms de ceux qui assurent la cargaison, ou des naviculaires, ou encore des mercatores. Enfin, une fois vidées, les amphores sont souvent réutilisées et l'on écrit alors au pinceau ou au charbon, ou l'on incise à la pointe dure de nourelles indications: des noms, des chiffres, des mesures...

Nous avons choisi de nous intéresser ici aux inscriptions peintes sur les amphores gauloises au moment de leur première utilisation. Ces indications concernent ce pourquoi l'amphore a été fabriquée : son contenu, la denrée transportée. Par leur complexité et leur variété, ces inscriptions traduisent mieux que les estampilles imprimées la maîtrise de l'écriture à main levée, mais aussi le rôle fondamental pour le commerce des denrées, de ce que l'on écrit et de ce que l'on donne à lire.

Le corpus des tituli picti sur amphores gauloises est resté longtemps fort maigre : on connaissait en tout et pour tout les inscriptions peintes de deux amphores venant de la région de Béziers, découvertes au Castro Pretorio à Rome et publiées par Dressel en 1879 dans le CIL, XV. À l'époque, et pendant de longues années encore, on ne savait rien d'autre sur les amphores gauloises. Grâce aux énormes progrès des fouilles sous-marines et terrestres, en France et dans les pays voisins, à ceux de la typologie et de l'épigraphie amphoriques durant les dernières décennies, on perçoit mieux désormais les productions gauloises. Il est même possible de dresser un corpus de leurs inscriptions peintes; il compte aujourd'hui plus de cent cinquante numéros et ne cesse de s'accroître. 


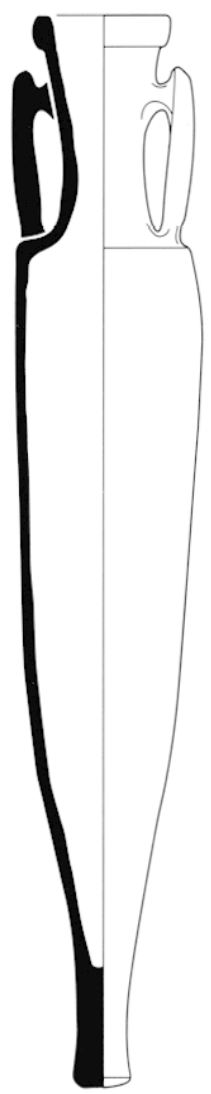

1

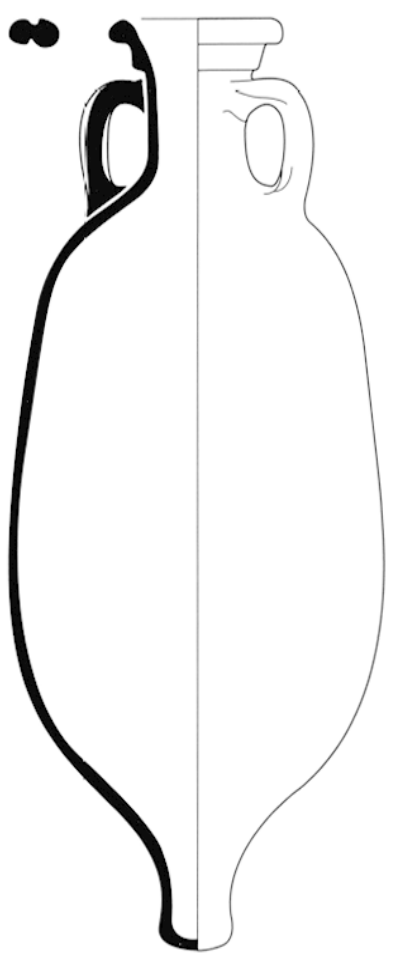

2

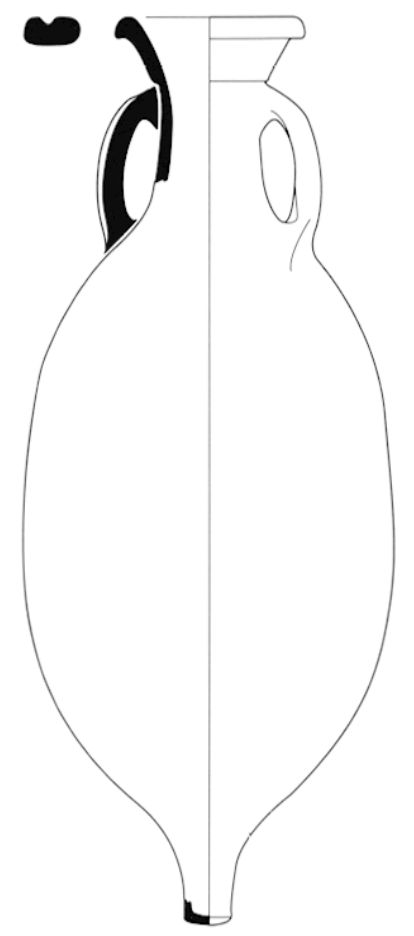

3

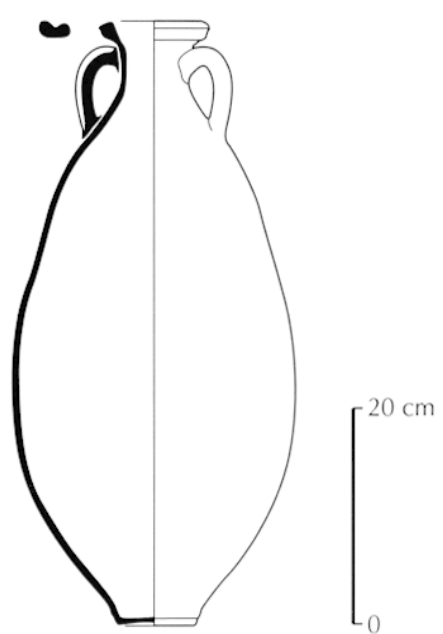

4

Fig. 79 - Principaux types d'amphores gauloises à sauces de poisson avec tituli picti : 1, Dressel 16; 2, Lyon 3A ou Dressel 10 similis ; 3, Lyon 3B ou Dressel 9 similis ; 4, Lyon 4.

L'origine des amphores gauloises n'est qu'exceptionnellement définie par les inscriptions qu'elles portent, comme la mention Baeterrense (de Béziers), sur les amphores qu'étudia Dressel, ou Antipolitanum (d'Antibes) sur d'autres, découvertes plus récemment. La recherche et l'étude des ateliers de potiers, de la typologie de leurs productions, mais aussi l'analyse physico-chimique des pâtes, ont permis de localiser en Gaule des zones de production, abondan- tes dans le sud et présentes un peu partout dans le reste du pays. On peut estimer que c'est au voisinage des officines que l'on remplissait les amphores et que l'on écrivait aussitôt les précieux renseignements sur la marchandise.

Parmi les cent cinquante-trois inscriptions dont nous disposons, la grande majorité concerne le vin: quatrevingt-treize; cinquante-deux concernent le poisson et huit les olives. Il n'y a aucune mention d'huile, et l'on est en droit de se demander si le sud de la Narbonnaise ne vendait pas son huile dans un autre type de contenant (des outres par exemple, comme c'était le cas dans l'Andalousie traditionnelle?).

Le corpus des inscriptions peintes reste, au demeurant, limité : d'une part, nous ne disposons pas de tituli picti pour l'ensemble de la gamme des amphores fabriquées en Gaule, parce qu'ils ont probablement disparu, d'autre part, les inscriptions sont souvent incomplètes.

\section{INSCRIPTIONS PEINTES SUR AMPHORES À POISSON}

Diverses sauces de poisson sont mentionnées à l'encre noire sur des amphores fabriquées en Gaule au Haut-Empire et sur une amphorette. Ces amphores sont de type Dressel 16, Lyon 3A (Dressel 10 similis), Lyon 3B (Dressel 9 similis), Lyon 4 et Augst 34 (fig. 79 et tabl. XV). Les indications qu'elles donnent correspondent en partie aux informations que l'on peut lire sur les amphores à poisson de Bétique - principal fournisseur de ces denrées depuis une période plus ancienne qui ont très certainement fourni un modèle d'inscription que l'on a simplifié en Gaule.

S. Martin-Kilcher, d'une part, R. Étienne et F. Mayet, d'autre part, ont proposé des schémas de lecture pour les tituli picti des amphores de Bétique à poisson et à huile (Martin-Kilcher, 1994, p. 420 et 2001 ; Étienne, Mayet, 2002, p. 21). Nous utiliserons celui que $\mathrm{S}$. Martin-Kilcher a adapté aux amphores gauloises contenant du poisson (fig. 80).

Une inscription principale est composée de plusieurs lignes horizontales disposées sur le col : en A, le produit ; en B, un ou plusieurs qualificatifs sur sa qualité, voire son origine ; en $C$ une quantité ; en D, le nom du commerçant. Une inscription secondaire, placée verticalement sur la droite près de l'anse, en F, indique un nom, souvent suivi d'un chiffre. 
Tabl. XV - Inscriptions peintes sur diverses formes d'amphores gauloises à poisson.

\begin{tabular}{|c|c|c|c|c|c|c|c|c|}
\hline \multicolumn{9}{|c|}{ AMPHORES À POISSON } \\
\hline Type & $\mathrm{N}^{\circ}$ & Découverte & $\begin{array}{c}\text { A } \\
\text { Produit }\end{array}$ & $\begin{array}{c}\text { B1 } \\
\text { Qualité }\end{array}$ & $\begin{array}{c}\text { B2 } \\
\text { Qualitatif }\end{array}$ & $\begin{array}{c}\text { C } \\
\text { Quantité }\end{array}$ & $\begin{array}{c}\text { D } \\
\text { Commerçant }\end{array}$ & $\stackrel{F}{\text { Inscription générale }}$ \\
\hline Dressel 16 & $\begin{array}{l}1 \\
2 \\
3 \\
4 \\
5 \\
6 \\
7\end{array}$ & $\begin{array}{l}\text { Londres } \\
\text { Rome } \\
\text { Augst P } 55 \\
\text { Ostie } \\
\text { Narbonne } \\
\text { Augst } \\
\text { Bretagne }\end{array}$ & $\begin{array}{l}\text { liquamen } \\
\text { liquamen } \\
\text { muria } \\
\text { muria } \\
\text { muria } \\
\text { garum } \\
\text { muria }\end{array}$ & $\begin{array}{l}\text { excellens } \\
\text { excellens } \\
\text { flos } \\
\text { flos } \\
\text { flos } \\
\text { flos }\end{array}$ & $\begin{array}{l}\text { Antipolitanum } \\
\text { Antipolitanum } \\
\text { Antipolitanum } \\
\text { Antipolitanum } \\
\text { Antipolitanum } \\
\text { Antipolitanum }\end{array}$ & N. XV & $\begin{array}{l}\text { L TETTI AFRICANI } \\
\text { LVM } \\
\text { LAVR } \\
\text { C. VIBI.SECVNDI }\end{array}$ & \\
\hline Lyon 3 & $\begin{array}{l}1 \\
2 \\
3 \\
4 \\
5\end{array}$ & $\begin{array}{l}\text { Koenigshoffen } \\
\text { Vindonissa } \\
\text { Mandeure } \\
\text { Mandeure } \\
\text { Vindonissa }\end{array}$ & $\begin{array}{l}\text { liquamen } \\
\text { muria } \\
\text { muria }\end{array}$ & & Antipolitanum & LXIII & R.V.F & $\begin{array}{l}\text { C IVL RNT XVI } \\
\text { M.V.P.VIII }\end{array}$ \\
\hline Lyon 3A & $\begin{array}{r}1 \\
2 \\
3 \\
4 \\
5 \\
6 \\
7 \\
8 \\
9 \\
10 \\
11 \\
12 \\
13\end{array}$ & $\begin{array}{l}\text { Lyon L } 16 \\
\text { Amiens } \\
\text { Koenigshoffen } \\
\text { Angers } \\
\text { Mayence } 37 \\
\text { Mayence } 38 \\
\text { Mayence } 39 \\
\text { Mayence } 40 \\
\text { Mayence } 41 \\
\text { Mayence } 42 \\
\text { Mayence } 43 \\
\text { Mayence } 44 \\
\text { Mayence } 76\end{array}$ & $\begin{array}{l}\text { garum } \\
\text { garum } \\
\text { garum } \\
\text { garum } \\
\text { garum } \\
\text { garum } \\
\text { garum } \\
\text { garum } \\
\text { garum } \\
\text { garum }\end{array}$ & $\begin{array}{l}\text { flos } \\
\text { flos } \\
\text { scombri } \\
\text { flos } \\
\text { flos } \\
\text { flos } \\
\text { flos } \\
\text { scombri } \\
\text { flos scombri } \\
\text { flos scombri }\end{array}$ & $\begin{array}{l}\text { A? } \\
P ? \\
\\
\text { LX? }\end{array}$ & 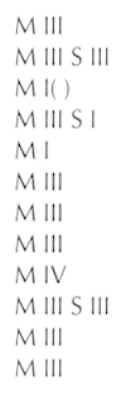 & MVR & $\begin{array}{l}\text { RVSTICI.IV } \\
\text { Q. VIRI. III } \\
\text { M.A.F.L XXXX } \\
\text { illisible } \\
\text { L Q AP P XXL } \\
\text { XX/C I G } \\
\text { IT.H.XV } \\
\text { C.A.C.X } \\
\text { A P IIIXV } \\
\text { ]XXII }\end{array}$ \\
\hline Lyon 3B & $\begin{array}{r}1 \\
2 \\
3 \\
4 \\
5 \\
6 \\
7 \\
8 \\
9 \\
10 \\
11 \\
12 \\
13 \\
14 \\
15 \\
16 \\
17 \\
18 \\
19 \\
20 \\
21 \\
22\end{array}$ & $\begin{array}{l}\text { Augst P } 42 \\
\text { Augst P } 45 \\
\text { Augst P } 52 \\
\text { Augst P } 54 \\
\text { Augst P } 40 \\
\text { Ausbourg } \\
\text { Mayence } 36 \\
\text { Lyon L 11 } \\
\text { Strasbourg ST } 178 \\
\text { Strasbourg ST } 165 \\
\text { Augst P } 41 \\
\text { Augst P } 43 \\
\text { Augst P } 44 \\
\text { Augst P } 46 \\
\text { Augst P } 47 \\
\text { Augst P } 48 \\
\text { Augst P } 49 \\
\text { Augst P } 50 \\
\text { Augst P } 51 \\
\text { Augst P } 53 \\
\text { Mayence } 46 \\
\text { Mayence } 77\end{array}$ & $\begin{array}{l}\text { garum } \\
\text { garum } \\
\text { garum } \\
\text { muria } \\
\text { muria } \\
\text { muria } \\
\text { muria } \\
\text { muria } \\
\text { muria } \\
\text { muria } \\
\text { muria } \\
\text { muria } \\
\text { muria } \\
\text { muria } \\
\text { muria } \\
\text { muria } \\
\text { muria }\end{array}$ & $\begin{array}{l}\text { excellens } \\
\text { excellens } \\
\text { excellens } \\
\text { excellens }\end{array}$ & $\begin{array}{l}\text { Hispana } \\
\text { AA? } \\
\text { Hispana } \\
\text { Hispana } \\
\text { Hispana } \\
\text { Antipolitanum } \\
\text { ()xtani } \\
\text { Hispana }\end{array}$ & $\begin{array}{l}\text { LXXI } \\
\text { LXX } \\
\text { LX() } \\
\text { LXXI } \\
\text { LXIIII } \\
(\text { )VI } \\
\text { XXIII } \\
\text { LXXII } \\
\text { LX() } \\
\text { LXX } \\
(L) X X \\
(L) X X \\
\\
\text { LXX } \\
\text { L() }\end{array}$ & $\begin{array}{l}\text { M.M. CL } \\
\text { ( )RSTI } \\
\text { ( )M( ) } \\
\text { M. M. CL } \\
\mathrm{T}(\text { ) } \\
\mathrm{T}(\text { ) L( ) M( ) } \\
\text { (M) M. CL } \\
\text { ( ) M. CL } \\
\text { M. M CL } \\
\text { ( )L( ) }\end{array}$ & $\begin{array}{l}\text { MASCVLI.V } \\
\text { (S)EX(ti). I( ) } \\
\text { G.P( ) C( ) II( ) } \\
\text { FRONTINVS V } \\
\text { L.V.F.X. } \\
\text { MVR } \\
\text { MASCVLI. V } \\
\text { M. T( ) M( ) } \\
\text { SEX. IV. } \\
\text { C. P( ) F( ) III } \\
\text { C. P( )C( )III } \\
\text { Q. S( ) L( ) ( ) } \\
\text { ()R S II( ) } \\
\text { M. E( )R( ) III } \\
\text { LEG( ) }\end{array}$ \\
\hline Lyon 4 & $\begin{array}{l}1 \\
2 \\
3\end{array}$ & $\begin{array}{l}\text { Augst } 84 \\
\text { Vindonissa } \\
\text { Lux }\end{array}$ & $\begin{array}{l}\text { liquamen } \\
\text { garum } \\
\text { illisible }\end{array}$ & $\begin{array}{l}\text { excellens } \\
\text { flos }\end{array}$ & & $\begin{array}{l}X X I \\
I I I I ?\end{array}$ & illisible & $\mathrm{S}(-) \mathrm{L}(-) \mathrm{SI}$ \\
\hline Augst 34 & 1 & Vindonissa & muria & flos & & MIIII SVIII & & \\
\hline Amphorette & 1 & Lyon L 17 & liquamen & & & & & APIC / G. RVFI \\
\hline
\end{tabular}




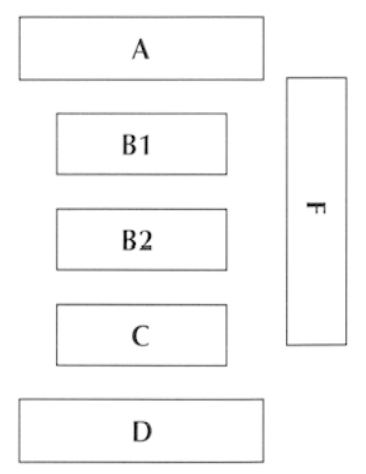

Fig. 80 - Schéma des inscriptions peintes sur les amphores gauloises à poisson (d'après Martin-Kilcher, 1994). Inscriptions horizontales : $A$, produit ; $B$, qualité et qualificatifs ; $C$, quantité ; $D$, nom du commerçant. Inscription verticale $: F$, noms et chiffres.

\section{LES DRESSEL 16}

Hautes et étroites, de faible capacité, elles correspondent à un type assez rare et relativement mal connu dont un atelier peut être attribué à Fréjus (Laubenheimer et al., 1992), un autre à Cannes (Laubenheimer, 1989) et un troisième à la région lyonnaise (Desbat et al., 1994). Six de ces amphores ont conservé sur le col une inscription à l'encre noire, en lettres capitales sur plusieurs lignes horizontales ${ }^{270}$. Il n'y a pas deux inscriptions identiques (tabl. XV) ; cependant, sans être stéréotypées, les formulations sont proches les unes des autres. Les premières lignes (A et B) sont consacrées au produit, à sa qualité et à son origine, la dernière (D), lorsqu'elle est conservée, indique les tria nomina d'un personnage qui a toutes chances d'être le marchand transportant le produit (fig. 81). Aucune inscription verticale sccondaire n'a été conservée. Les sauces sont variées : du liquamen qualifié d'excellens, ou du garum et de la muria qui sont flos, c'est-à-dire vierges (Étienne, Mayet, 2002, p. 50). Les mots employés sont ceux-là mêmes que l'on utilise pour les produits de Bétique. En revanche, on n'indiquera ici ni l'âge des sauces ni leur quantité ${ }^{271}$, tandis que leur origine paraît régulièrement mentionnée lorsque l'inscription est complète. Soulignons que le qualificatif est unique pour les trois produits : Antipolitanum (d'Antibes) ! S'agit-il réellement du lieu de fabrication précis du produit? En réalité, ce terme ne semble pas exclusif de la ville d'Antibes puisqu'il apparaît sur des amphores fabriquécs ailleurs: l'une à Fréjus et une autre, de type Lyon 3, produite dans un autre atelier provençal (Laubenheimer et al., 1992 ; Picon, 1992). Ainsi, l'appellation Antipolitanum doit-elle être comprise comme un label « à la façon d'Antibes " et recouvrir au moins unc large zone géographique de la côte provençale.

Ces produits de grande qualité, emballés dans des amphores de petites capacités (moins de 9 litres), sont diffusés à longue distance sur des marchés remarquables: Ostie, Rome,

270. L'inscription d'Ostic se trouvant sur un col incomplet, cela permet d'hésiter sur la forme: Dressel 16 ou Dressel 2/4.

271. Le chiffre indiqué sur l'amphore de Narbonne correspondrait à un numéro et non à une contenance d'après liou, 1993.

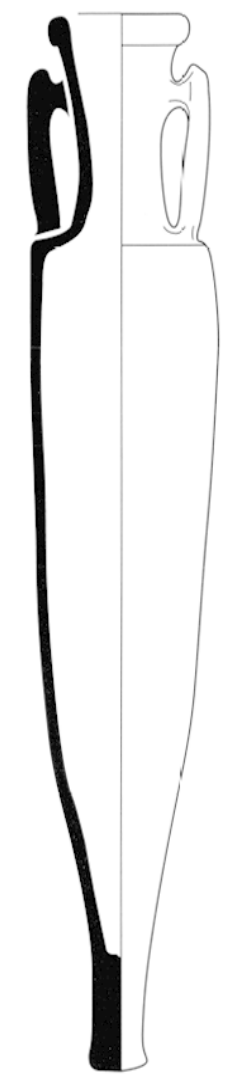

\section{$\operatorname{sic}^{1} x h$}

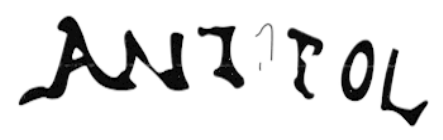

$\therefore \times c$

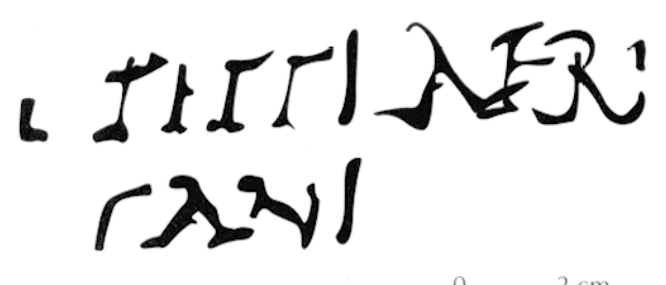

Fig. 81 - Exemple de titulus pictus sur une amphore gauloise à poisson Dressel 16, découverte à Londres el fabriquée à Antibes : Liqua(men), Antipol(itanum) I Exc(ellens) I L(ucii) Tet(i) Afri I cani (d'après Laubenheimer dir, 1992).

Augst et Londres, probablement à l'époque flavienne, comme l'indiquent les contextes d'Augst et de Londres.

\section{LES DRESSEL 10 SIMILIS ET DRESSEL 9 SIMILIS}

Ces amphores ovoïdes, repérées pendant longtemps comme inclassables (Ettlinger, 1977 ; Paunier, 1981 ; Jeannin, Laubenheimer, 1989), sont désormais attribuées essentiellement à la région lyonnaise d'après leur composition (Schmitt, 1988 ; Dangréaux et al., 1992). On distingue un type plus ancien Dressel 10 similis ou Lyon 3A ${ }^{272}$, daté dans les fouilles lyonnaises entre le début du $\mathrm{I}^{\mathrm{er}} \mathrm{s}$. et l'époque de Claude-Néron, auquel succède un type 3B (Dressel 9 similis) bien distribué sous les Flaviens (Desbat, Dangréaux, 1997).

Treize amphores Dressel 10 similis, Lyon 3A, portent régulièrement sur leur col deux types d'inscriptions en lettres capitales peintes en noir ${ }^{273}$. L'une, frontale, disposée sur plusieurs lignes horizontales, décrit le produit, sa qualité et sa

272. I a typologic de ces amphores n'a pas encore été définic de façon tout a fait rigoureuse et univoque si bien que certains classements sont ambigus (Martin-Kilcher, 1994; Desbat, Dangréaux, 1997).

273. I.es amphores de Kocnigshoffen et d'Angers sont ici attribuées à ce groupe dont elles ont la lève caractéristique et le même type d'inscription. La séparation des groupes $3 \mathrm{~A}$ et $4 \mathrm{~A}$ proposéc par A. Desbat et B. Dangréaux en 1997 manque de précision. 
quantité. L'autre, perpendiculaire, à droite près de l'anse, indique le nom d'une personne. La désignation du produit on abrégé à la première ligne horizontale, en $\mathrm{A}$, est, pour les exemples lisibles, du (arum), qualifié six fois de $F(l o s)$, deux fois de $S C$ (ombri) (maquereau) et deux fois de flos scombri, sans connotation d'origine. Les lettres $\mathrm{G}$ et F ou SC sont grandes et peu soignées. La quantité, évaluée en modii et sextarii, est mentionnée à la ligne inférieure $(C)$, elle oscille autour de trois modii, soit vingt-six litres, la contenance de l'amphore (fig. 82).

L'inscription verticale latérale, inscrite sans doute dans un deuxième temps (en F), indique un nom (contrôle?), suivi d'un chiffre ou d'un nombre que l'on n’interprète pas.

\section{LES AMPHORES LYON 3B}

Elles sont plus nombreuses en général et il n'est pas étonnant que le corpus des marques peintes soit plus fourni : vingt-deux excmplaires. L'écriture en capitales est régulière et soignée. On retrouve le même schéma d'unc inscription frontale horizontale et d'une autre verticale à droite (fig. 82).

L'inscription horizontale se développe sur trois ou quatre lignes. La première, en $\mathrm{A}$, indique la nature du produit : de la muria essentiellement (quatorze fois), plus rarement du garum (trois fois). Le qualificatif d'origine suit immédiatement le nom du produit, sur la même ligne ou sur la ligne suivante, en B : Hispana (cinq fois) ou Antipolitanum (une fois). Vient ensuite la désignation facultative de la qualité (en B) : toujours excellens, aussi bien pour le garum que pour la muria. À la ligne suivante, en $\mathrm{C}$, comme on pouvait s'y attendre, se trouve l'indication d'une quantité qui oscille autour du chiffre LXX pour une douzaine d'exemples et dont l'unité n'est pas précisée. Il pourrait s'agir de livres, ce serait alors le poids du contenu, équivalent à environ 23 litres (Martin-Kilcher, 1994, p. 420). En dernière ligne, apparaît le nom du commerçant désigné par ses tria nomina. À Augst, un certain M. M. CL. commercialise du garum et de la muria Hispana.

Verticalement, en F, un cognomen ou des tria nomina inscrits lors d'un probable contrôle sont suivis d'un chiffre toujours mystéricux. C'est le même système que pour les Lyon $3 \mathrm{~A}$.

En revanche, pour les $L y$ on $3 B$, on remarque une évolution de l'inscription horizontale (en A, B et C). La calligraphie est différente, plus soignée et régulière, la qualité des produits change aussi, on passe du garum flos au garum et à la muria excellens, qui sont, de surcrô̂t, Hispana ou Antipolitanum. Enfin, l'évaluation de la quantité du produit n'est plus faite dans la mêtme unité de mesure, s'il s'agit ici de poids. Tout se passe comme s'il y avait une sorte de normalisation.

La présence du terme Antipolitanum sur une amphore Lyon 3B fabriquée dans la région de Lyon (Desbat $e t$ al., 1994) conforte l'idée qu'il s'agit là d'un label peut-être sans attache régionale stricte, à moins que le produit provençal n'ait été mis en amphores à Lyon !

Le qualificatif d'Hispana est curicux parce qu'il fait référence à un pays et non à une cité, comme c'est le plus souvent le cas pour les sauces de poisson de Bétique, de Gadès ou de Malaga, par exemple. Ce terme générique caractérise plus certainement une recette "à la façon de " (comme le suggérait déjà Bohn, en 1926) qui peut avoir été fabriquée
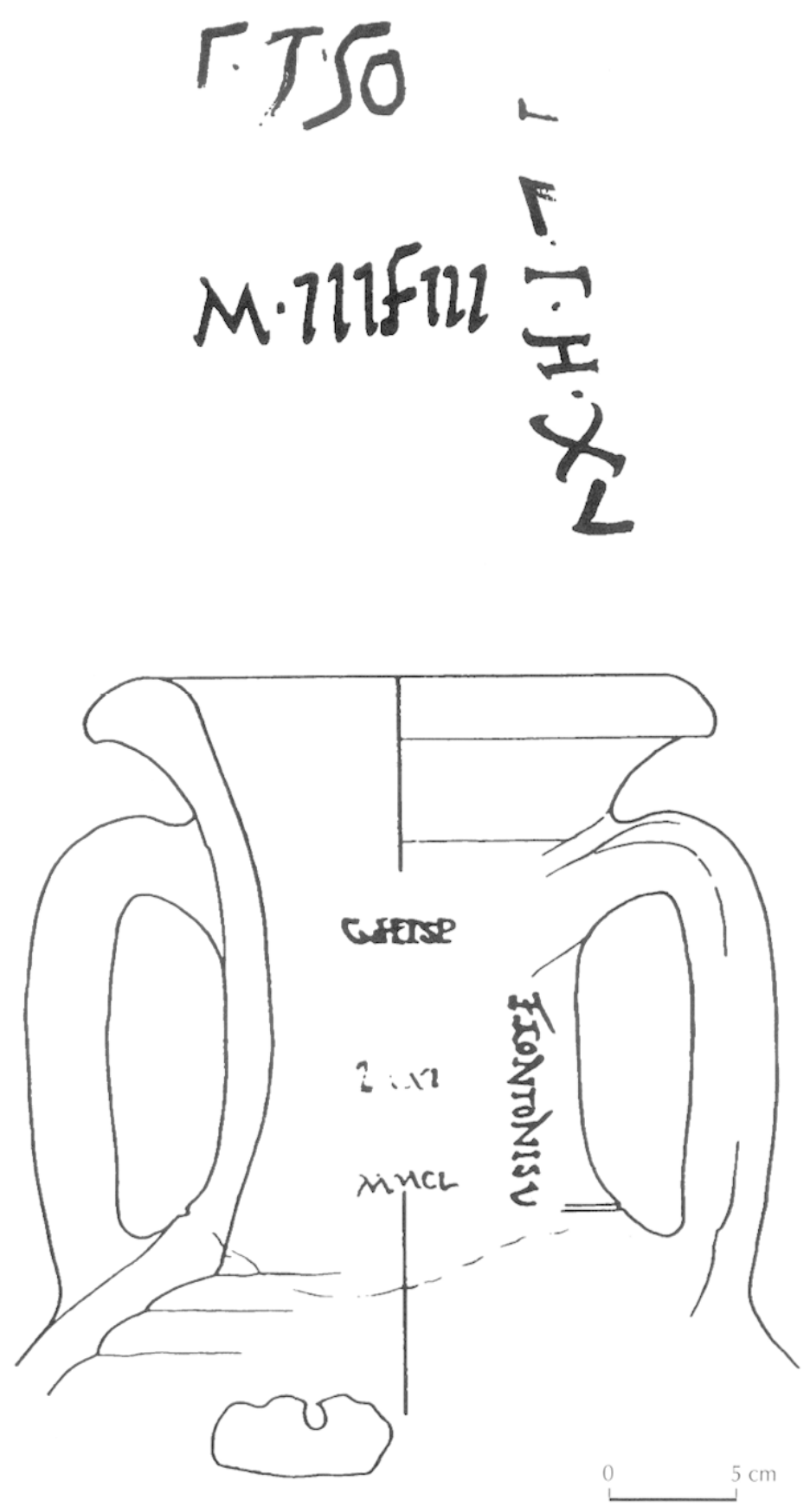

Fig. 82 - Exemples de tituli picti sur des amphores à poisson : 1, Lyon 3A, Dressel 10 similis découverte à Mayence, inscription horizontale: $\mathrm{G}$ (arum) F(los) SC(ombri) I M(odii) III et inscription verticale : (--) T.H.XV (d'après Ehmig, 2003, titulus 42);2, Lyon 3B, Dressel 9 similis découverte ì Augst, inscription horizontale: G(arum) HISP(anum) I LXXI । M.M.CL et inscription verticale: FRONTINVS V (d'après Martin-Kilcher; 1994, fig. 187).

n'importe où, y compris en Gaule méridionale. Le produit importé en vrac à Lyon aurait été conditionné là-bas en amphores (Desbat, Dangréaux, 1997 ; Martin-Kilcher, 2002). Venait-il d'Espagne? Rien n'est moins sûr ! En effet, on importait bien à Lyon, et en nombre, à la même époque, comme dans le reste de la Caulue, des amphores de Bétique à 
sauces de poisson aux formes et aux inscriptions caractéristiques dont l'origine ne laisse pas de doute.

Si l'on compare les termes Antipolitanum et Hispana, on est frappé par le parallélisme qu'il y a dans leur usage : qualifiant le même produit, la muria excellens, ils paraissent interchangeables; ils sont placés à la première ou à la deuxième ligne de l'inscription, dans une zone réservée à la description de la qualité du produit.

Antipolitanum est inscrit sur des amphores gauloises de type Dressel 16 ou Lyon 3, fabriquées soit en Provence (à Fréjus et ailleurs, cf. Picon, 1992), soit dans la région lyonnaise, c'est le cas d'une Lyon 3B découverte à Augst (Desbat et al., 1994). Hispana est peint sur des amphores gauloises de forme parfaitement identique (Lyon 3B, et seulement sur celles-ci, dans l'état actuel de nos connaissances), d'origine lyonnaise et pourquoi pas un jour provençale, si le terme n'évoque qu'une recette! En effet, la forte cohérence qui existe entre produits et emballages portant le qualificatif soit Hispana, soit Antipolitanum, conduit à envisager l'hypothèse d'une source de production commune qui pourrait être gauloise et provençale : les sauces étant acheminées vers Lyon en amphores, dès le départ, ou en vrac et mises en amphores à l'arrivée, dans des emballages toujours identiques, caractéristiques des produits gaulois.

\section{LES AMPHORES LYON 4 OU AUGST 17}

De petit module, avec un fond plat, elles sont plus rares et sont attribuées au groupe lyonnais $\mathrm{du}^{\mathrm{er}} \mathrm{s}$. Une inscription frontale peinte en capitales noires sur une amphore d'Augst (Martin-Kilcher, 1994, fig. 156), estampillée C.L( ) ANT( ), ne comporte que deux lignes : en A, le nom du produit, LIQ(amen) et en B, sa qualité, EXC(ellens) (fig. 83). Signalons aussi un autre col découvert à Vindonissa, qui pourrait être unc Lyon 3A d'après S. Martin-Kilcher (1994, p. 381), et une Lyon 4 d'après A. Desbat et B. Dangréaux (1997, p. 102), qui porte en A, G(ari) $F(l o s)$ et en $\mathrm{C}$, le chiffre XXI. Enfin, l'inscription de l'amphore Lyon 4 de Lux, dans la vallée de la Saône, est de lecture difficile.

\section{LES AMPHORES AUGST 34}

Attribuées à la côte provençale par S. Martin-Kilcher (1994, p. 415), elles sont rares et datées d'Auguste au II" s. Un exemplaire complet trouvé à Nyon contient 44,2 litres. Un col provenant de Vindonissa porte l'inscription $M V R(i a e) F(l o s)$, comme les petites Dressel 16, et sa capacité est indiquée en modii et sextarii, comme sur les Lyon $4 \mathrm{~A}$.

\section{L'AMPHORETTE DE LYON}

Enfin, il semble que l'on doive considérer comme gauloise une amphorette de Lyon portant le titulus: IIQ(amen) / APIC / G.RVFI (Desbat et al., 1987, p. 157).

En définitive, on reste surpris par la variété de ces amphores gauloises à poisson : différences dans leurs formes, nous en avons recensé cinq, et différences dans leurs capacités (de 9 litres, ou moins, à 44 litres) ! Contrairement à ce que l'on aurait pu penser, les types de produits et leur qualité ne sont pas

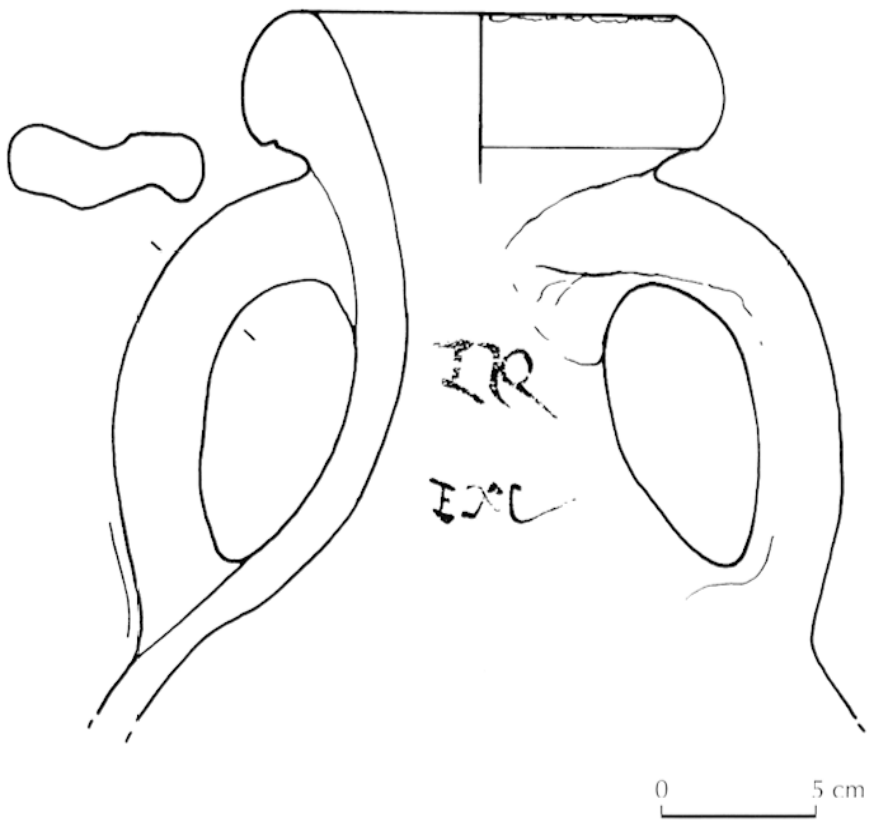

Fig. 83 - Exemple de titulus pictus sur une amphore Lyon 4 ou Augst 17, découverte à Augst : LIQ(uamen) EXC(ellens) (d'après Martin-Kilcher, 1994, fig. 156).

associés au volume de marchandise transportée. Les petites Dressel 16, comme les petites Lyon 4, transportent aussi bien du liquamen que de la muria ou du garum et ce sont les mêmes produits que l'on retrouve dans les fortes Lyon 3. Il apparaît néanmoins, au vu de notre corpus (cf. infra, p. 166-171), que les Dressel 16 sont surtout spécialisées dans les produits d'Antipolitanum et que les produits qualifiés d'Hispania ne concernent que les amphores Lyon 3B de l'époque flavienne.

Au regard des amphores à saumures et sauces de Bétique, les formes gauloises se distinguent nettement avec, sans doute, une probable inspiration typologique hispanique pour les Lyon 3, mais guère pour les autres. Au reste, les tituli picti qu'elles portent sont plus courts, certains qualificatifs hispaniques ne semblent pas être utilisés, comme summum ou l'âge des sauces, par exemple. En revanche, la désignation des produits : garum, muria ou liquamen reprend les mots latins habituels, sans originalité particulière. Une enquête complémentaire est à mener sur les commerçants et les contrôles. Il est clair que maîtriser la lecture et l'écriture était indispensable pour qui voulait vendre ou acheter les sauces de poisson mises en amphores en Gaule, tant sont variés les produits et les emballages.

\section{INSCRIPTIONS PEINTES SUR AMPHORES À OLIVES}

I.cs amphores gauloises de type Augst 21 ont été fabriquées à Lyon et dans la vallée du Rhône (Desbat, Dangréaux, 1997, p. 79). Adaptation gauloise ${ }^{274}$ à l'époque flavienne et au II's $^{\prime}$.

274. P. R. Sealey et P. A. Tyers estiment que ces amphores pourraient avoir eu plusieurs origines (Sealcy, Tyers, 1989). 
Tabl. XVI - Inscriptions peintes sur amphores gauloises à olives Augst 21.

\begin{tabular}{|c|c|c|c|c|c|c|c|c|}
\hline \multicolumn{9}{|c|}{ AMPHORES À OLIVES } \\
\hline Type & $\mathrm{N}^{\circ}$ & Découverte & $\begin{array}{c}\text { A } \\
\text { Produit }\end{array}$ & $\begin{array}{c}\text { B1 } \\
\text { Qualité }\end{array}$ & $\begin{array}{c}\text { B2 } \\
\text { Qualité }\end{array}$ & $\begin{array}{c}\text { C } \\
\text { Quantité }\end{array}$ & $\begin{array}{c}\text { D } \\
\text { Commerçant }\end{array}$ & $\stackrel{F}{\text { Inscription générale }}$ \\
\hline Augst 21 & $\begin{array}{l}1 \\
2 \\
3 \\
4 \\
5 \\
6 \\
7 \\
8\end{array}$ & $\begin{array}{l}\text { Londres } \\
\text { Colchester } \\
\text { Vechten } \\
\text { Londres } \\
\text { Augst } \\
\text { Nyon } \\
\text { Vindolanda } \\
\text { Mayence }\end{array}$ & $\begin{array}{l}\text { olivae } \\
\text { olivae } \\
\text { olivae } \\
\text { olivae } \\
\text { olivae } \\
\text { olivae } \\
\text { olivae } \\
\text { olivae }\end{array}$ & $\begin{array}{l}\text { albae } \\
\text { albae } \\
\text { exdulci } \\
\text { ex dulci } \\
\text { albae } \\
\text { albae }\end{array}$ & excellens & $\mathrm{CCL}$ & $\begin{array}{l}C L() P() \\
C L() A() / V I A N V ? \\
M C I R()\end{array}$ & $\mathrm{L}() \mathrm{L}() \mathrm{LVCCI} \mathrm{L}() \mathrm{C}() \mathrm{H}()$ \\
\hline
\end{tabular}

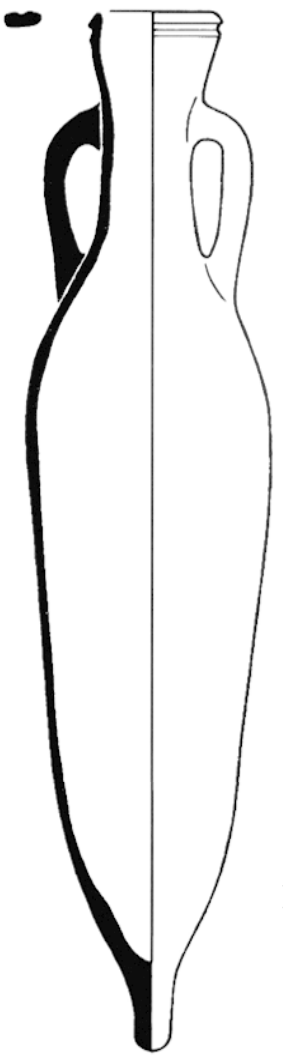

Fig. 84 - Exemple de titulus pictus sur une amphore à olives Augst 21, découverte. à Augst : (o)l(ivae) ( ) | ex I dul(ci) (d'après Martin-Kilcher, 1994, fig. 169).

d'un modèle plus ancien, Haltern 70, fabriqué en Bétique, il est distribué vers les provinces du nord: Germanie, Bretagne et Gaule belgique (Martin-Kilcher, 1994, p. 391). Ce sont des amphores assez rares dont les tituli picti indiquent qu'elles contenaient, comme les Haltern 70, des olives (en A) dont on sait (en B) qu'elles sont parfois vertes ou mêlées à du vin doux qui peut être excellens (fig. 84 et tabl. XVI). Un chiffre qui peut correspondre à une quantité est une seule fois conservé. Le nom du commerçant (en D) apparaît à plusieurs reprises. Une inscription verticale est également présente, mais rarement conservée. Elle comprend des noms. On retrouve ici encore un type d'inscription qui s'inspire directement de celui de Bétique, mais le produit est différent puisque les olives sont conservées dans du vin doux et non dans du vin cuit comme le defrutum ou la sapa des Haltern 70. On peut s'interroger sur l'origine des produits transportés : olives du midi de la Gaule ou olives de la péninsule Ibérique?

\section{INSCRIPTIONS PEINTES SUR AMPHORES VINAIRES}

Plus nombreuses sont les amphores vinaires en général, ainsi que les tituli picti conservés: quatre-vingt-huit sont ici rassemblés (tabl. XVII). Ils correspondent à des amphores de types Dressel 2/4 gauloise et Gauloise 3, 4, 5 et 7. L'inscription principale, sur le col, composée de plusieurs lignes horizontales, suit un schéma qui n'est pas très éloigné des précédents. Toutefois, à la différence des sauces de poisson ou des olives, le produit lui-même, le vin, n'est généralement pas mentionné ; il est simplement désigné par des qualificatifs qui concernent, en $B$, le cépage, la qualité, l'âge, l'origine, parfois ; en $C$, une quantité et en $\mathrm{D}$ apparaît, comme à l'accoutumée, le nom du commerçant. Très rarement, trois fois seulement, est indiquée une date consulaire. En oblique, près de l'anse, à droite, une inscription secondaire signale le plus souvent un nom, parfois des chiffres ou d'autres indications encore.

\section{LES DRESSEL 2/4}

Sur deux exemplaires du Castro Pretorio à Rome (tabl. XVII, $\mathrm{n}^{\text {os }} 1$ et 2), E. Dressel a découvert les deux premières inscriptions gauloises peintes qui sont aussi les plus anciennes, datées de la période augustéenne (CIL, XV, 4542 et 4543 ; Dressel, 1879, p. 63). Elles sont tout à fait extraordinaires à plus d'un titre. En effet, ce sont les seules, parmi toutes les amphores gauloises, sur lesquelles on a écrit en blanc et non en noir. De plus, la première parle à la première personne, ligne A : sum (je suis), formule exceptionnelle qui ne répond à 


\begin{tabular}{|c|c|c|c|c|c|c|}
\hline & 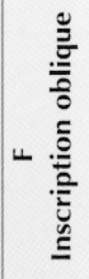 & $\overrightarrow{2}$ & & & 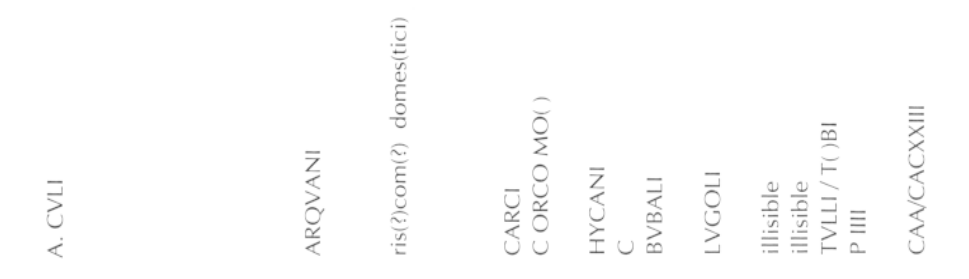 & 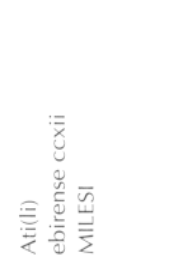 \\
\hline \multirow{11}{*}{ 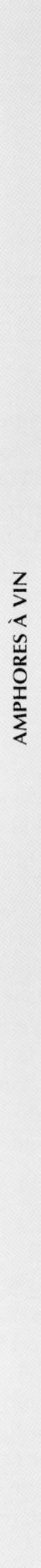 } & ○ & 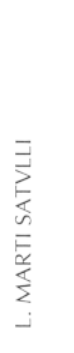 & & & 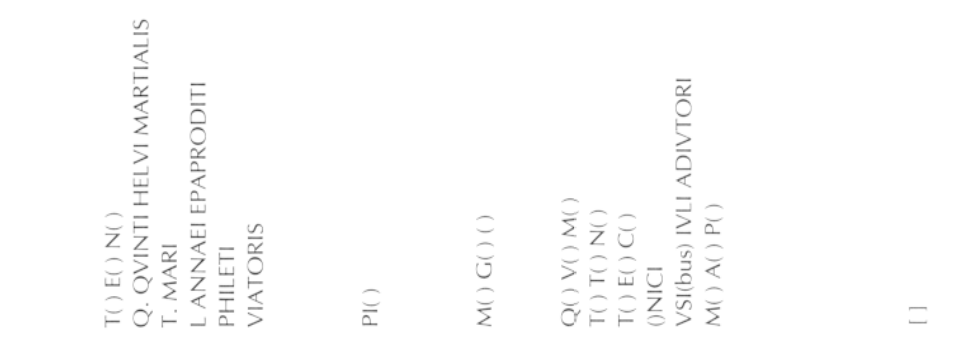 & 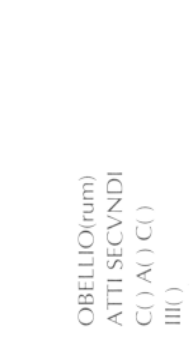 \\
\hline & u & & & & 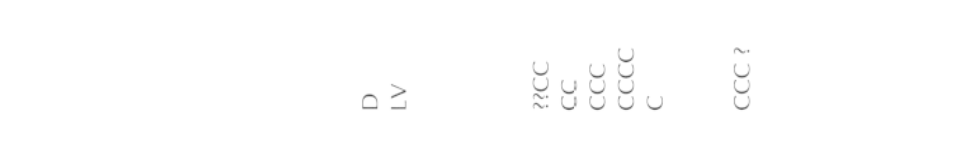 & $\bar{x}$ \\
\hline & 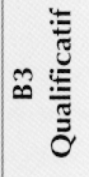 & 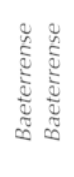 & $\frac{\tilde{g}}{\bar{E}}$ & & 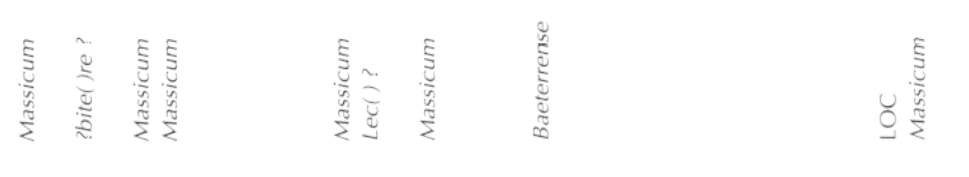 & 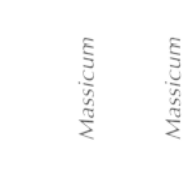 \\
\hline & 今 : & $>$ & & & $\equiv$ & \\
\hline & ๙ & & & & 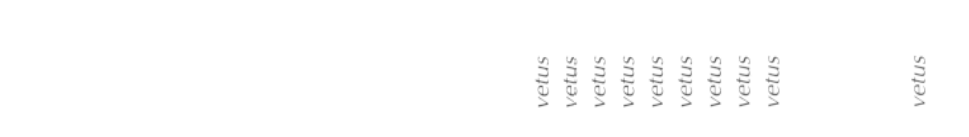 & 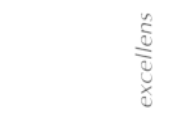 \\
\hline & 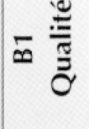 & 胥 & 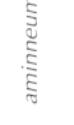 & 密 密 & 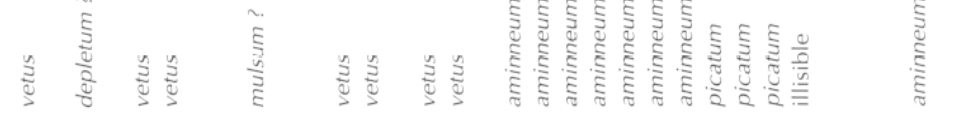 & \\
\hline & $\stackrel{\frac{\pi}{\pi}}{\varrho}$ & & 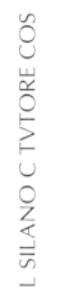 & & & \\
\hline & $\ll \frac{\bar{E}}{\frac{\bar{E}}{2}}$ & 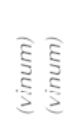 & ह气 & ह气 & 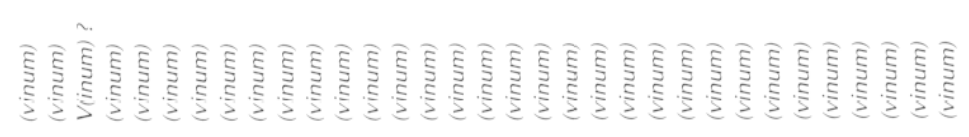 & 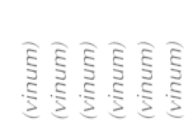 \\
\hline & 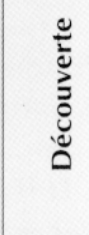 & 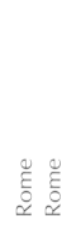 & 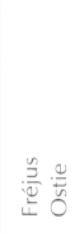 & 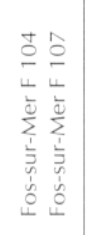 & 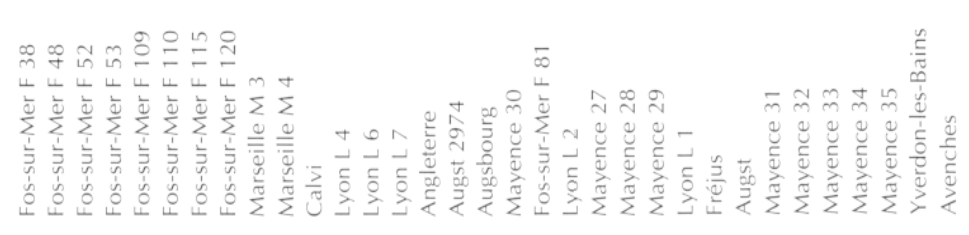 & 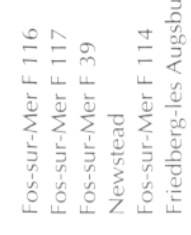 \\
\hline & $\stackrel{\circ}{z}$ & $-n$ & $-\lambda$ & $-N$ & 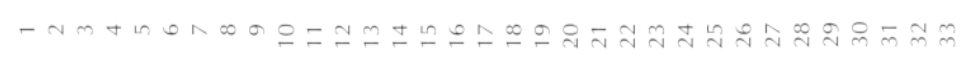 & $-N m+i n \in$ \\
\hline & $\sum_{2}^{\circ}$ & 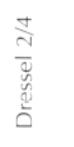 & 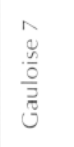 & 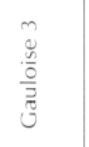 & 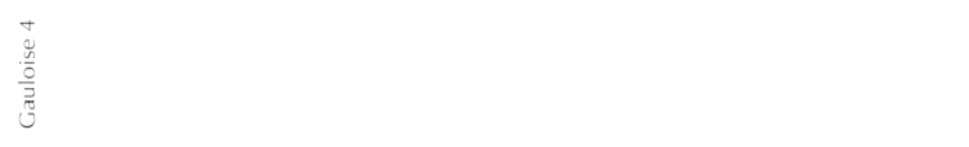 & 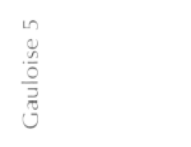 \\
\hline
\end{tabular}




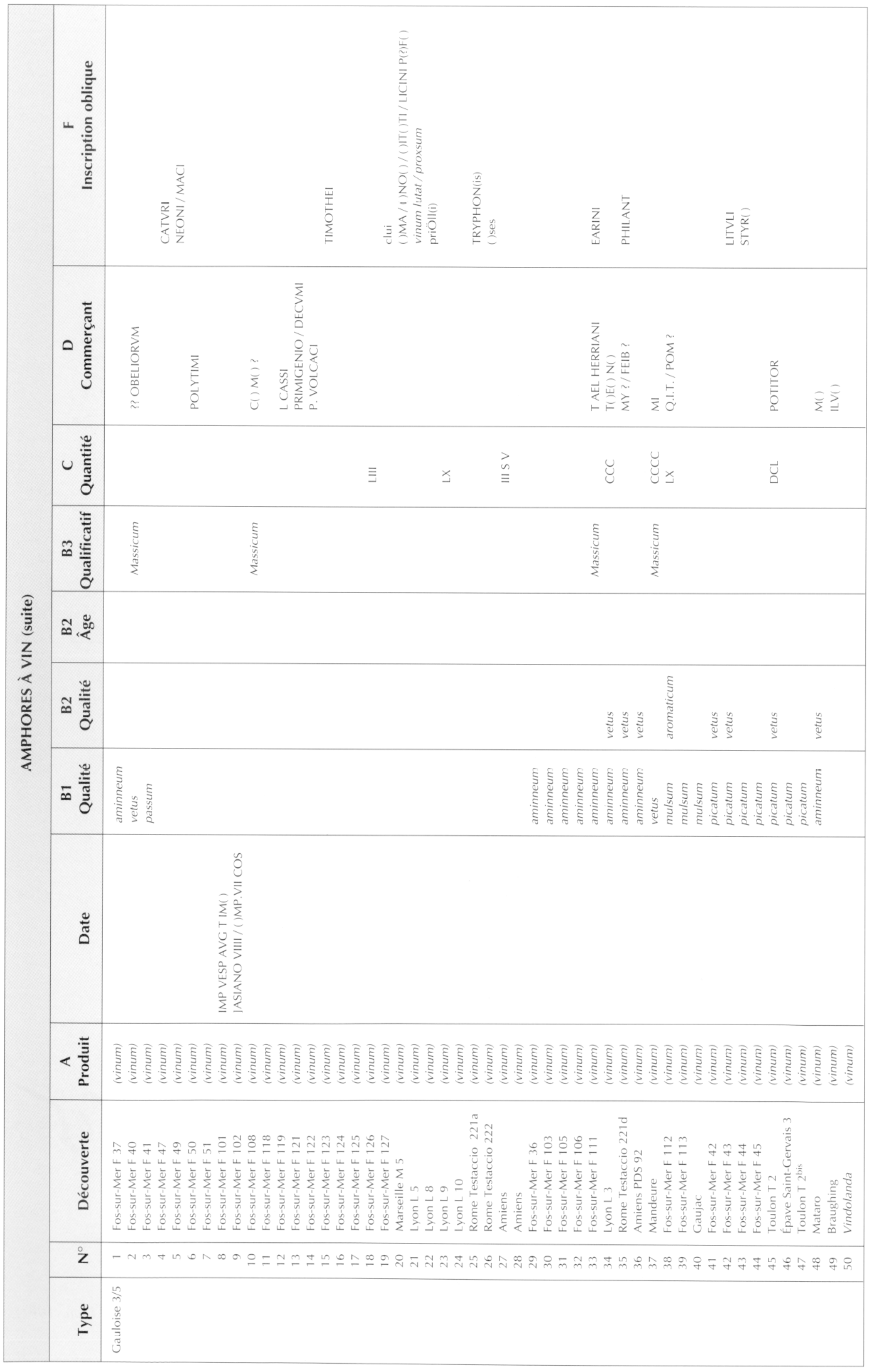



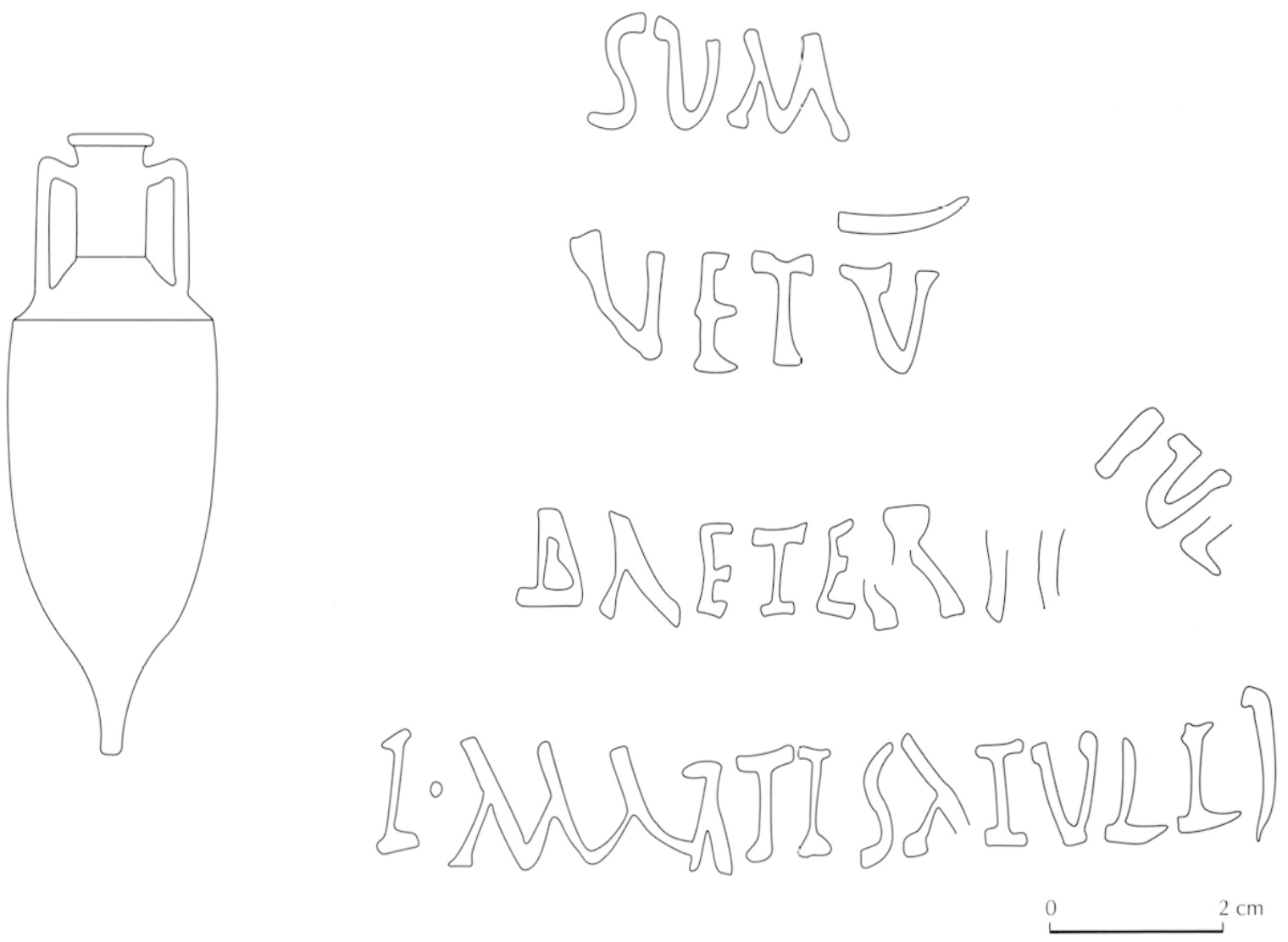

Fig. 85 - Exemple de titulus pictus sur une amphore Dressel 2/4 découverte au Castro Pretorio (Rome) : SVM I VET(us) V I BAETERERRE(NSE). L(ucii) MARTI SATVLLI (d'après Dressel : CIL, XV, 4542).
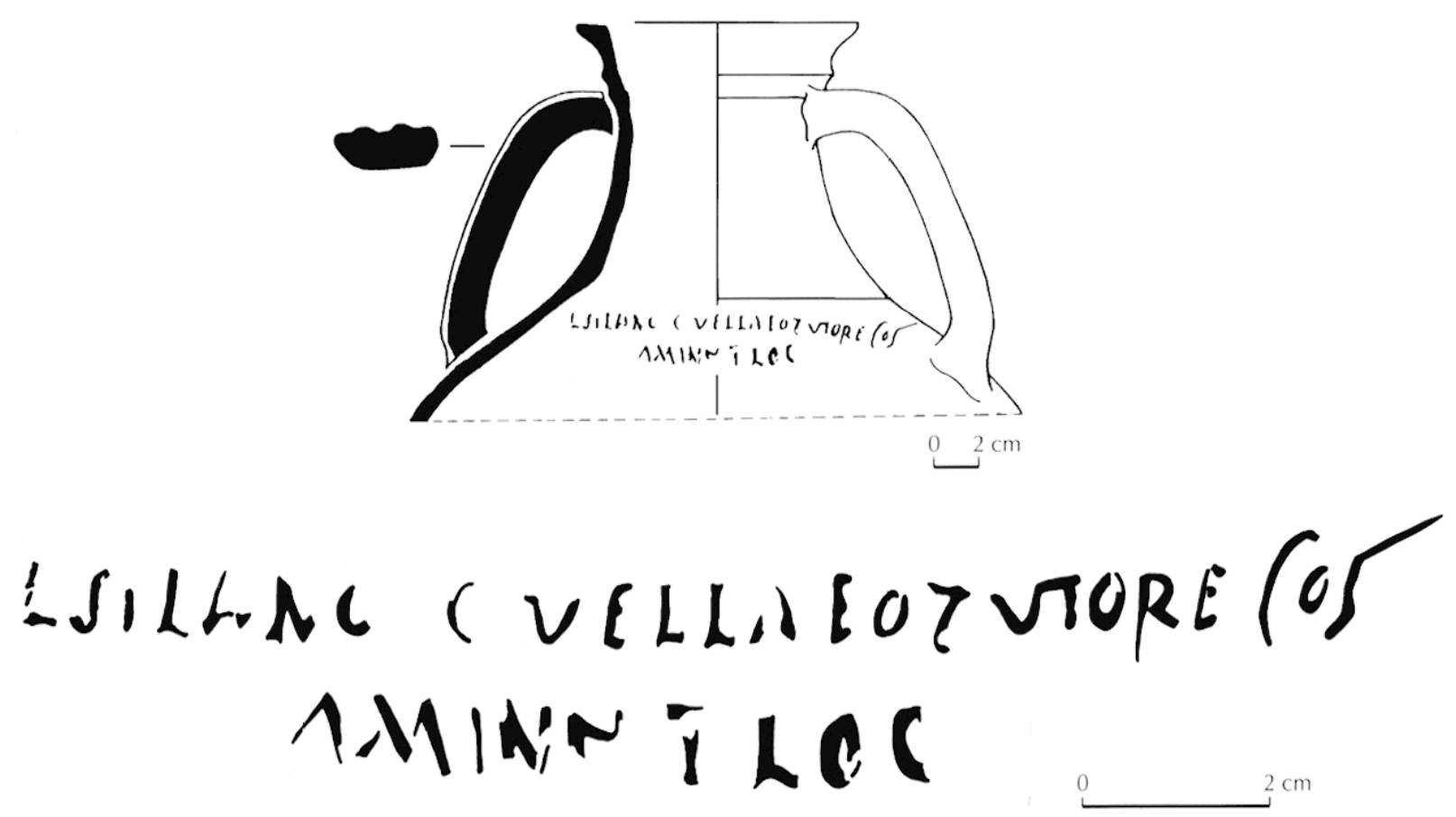

Fig. 86 - Exemple de titulus pictus sur une amphore Gauloise 7 découverte à Frëjus :

L(ucio) (Iunio) SILANO C(aio) VELLAEO TVTORE CO(n)S(ulibus) I AMINN(cum) T LOC (d'après Liou, 1991). 

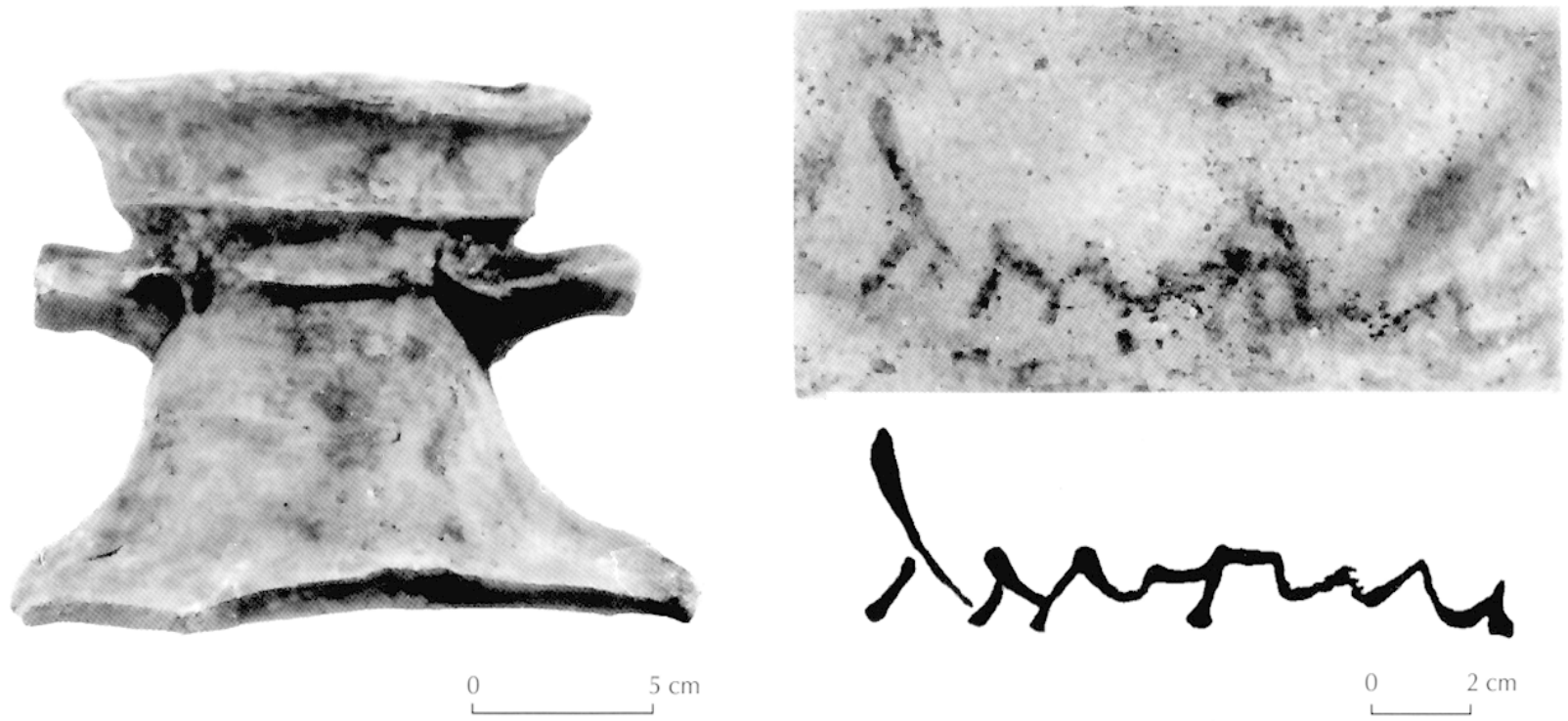

Fig. 87 - Exemple de titulus pictus sur une amphore Gauloise 3 découverte à I'os-sur-Mer: Aminn(eum) (d'après Liou, 1987, F 104).

aucun modèle connu (fig. 85). Elle contient du vin, qualifié comme souvent de vetus (vieux), dont l'âge est indiqué : $V$, cinq ans, suivant une formule fréquemment employée sur les amphores italiques, mais rare sur les tituli gaulois que nous connaissons. Le vin est qualifié aussi de Baeterrense, de Béziers. À la ligne D, apparaissent les tria nomina du commerçant. Verticalement, on lit encore, comme généralement, un nom, d'une autre écriture probablement postérieure. Sur la seconde amphore, apparaissent deux qualificatifs : le vin est également Baeterrense et cette fois il est blanc, cas unique parmi les tituli picti gaulois. La région de Béziers est bien connue pour ses nombreux ateliers d'amphores, on y fabriquait notamment des Dressel 2/4 (Laubenheimer, 1985).

\section{LES GAULOISE 3, 4, 5, 7}

Parmi les premiers tituli gaulois, on note aussi deux amphores de forme Gauloise 7 (tabl. XVII, nos 1 et 2). L'une, découverte à Fréjus (Liou, 1991), porte une date consulaire, 28 apr. J.-C., ce qui est rarissime pour une amphore vinaire gauloise (fig. 86). Elle contient de l'aminneum. L'autre, découverte à Ostie, porte l'indication Cirt(a) ou Cirl(ensis) que l'on explique mal (Carta et al., 1978).

Deux cols découverts à Fos-sur-Mer sont attribuables au type Gauloise 3 (tabl. XVII, $\mathrm{n}^{\text {os }} 1$ et 2 ). Ils indiquent tous deux du vin aminneum (fig. 87).

Pour les six Gauloise 5 connues (tabl. XVII, $n^{\text {os }} 1$ à 6 ), on apprend que l'une contient du picatum excellens (fig. 88), deux autres du Massicum.

Les Gauloise 4, amphores du grand commerce par excellence, de la seconde moitié du $\mathrm{I}^{\mathrm{er}} \mathrm{s}$. au début du $\mathrm{III}^{\mathrm{e}}$ s., sont, comme l'on pouvait s'y attendre, les plus nombreuses : trentedeux exemplaires (tabl. XVII). Elles offrent des combinaisons de données particulièrement intéressantes.
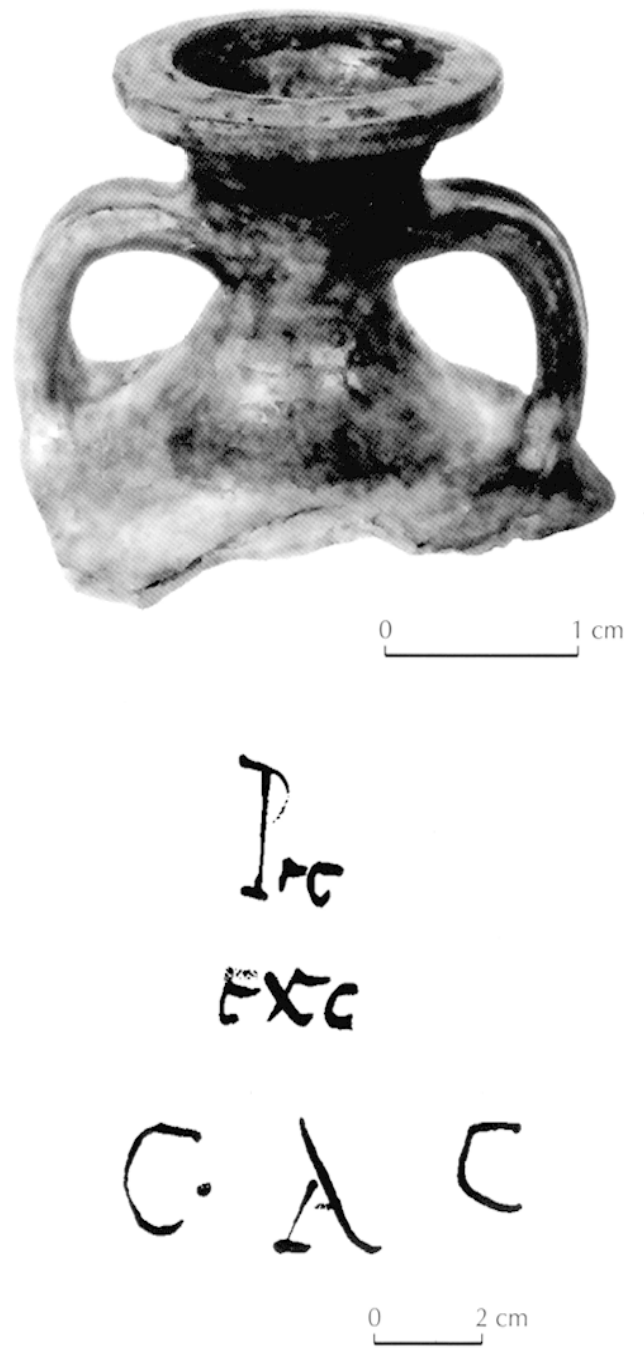

Fig. 88 - Exemple de titulus pictus sur une amphore Gauloise 5 découverte à Fos-sur-Mer : PIC(atum) I EXC(ellens) I C.A.C (d'après Liou, 1987, F 114). 
Leur contenu se compose de divers vins ${ }^{27,}$, de l'aminneum vetus surtout, mais aussi du picatum vetus, peut-être même du mulsum et du depletum. Parfois, le vin est simplement qualifié de vetus. Il n'y a d'ailleurs pas d'autre qualificatif de qualité. On voit tout de suite que le même emballage sert à transporter plusieurs sortes de vins. Ils correspondent à des cépages ou à des types de vinification connus en Italie et développés en Gaule.

Deux qualificatifs qui font allusion à une origine géographique sont utilisés: Baeterrense, de Béziers, déjà connu sur Dressel 2/4, apparaît sur une Gauloise 4, associé à de l'aminneum vetus (fig. 89 et tabl. XVII, n 19 ). La région de Béziers est particulièrement riche en officines d'amphores Gauloise 4 (Mauné, 2001).

Le terme Massicum désigne une zone précise de la Campanie, le Massique (Liou, 1987, p. 74). Il est plus fréquent (cinq occurrences sur Gauloise 4, voir fig. 89 et tabl. XVI, $\mathrm{n}^{\mathrm{os}} \mathrm{l}$, $5,6,12,15)$ et régulièrement associé au qualificatif vetus ${ }^{276}$. Il est aussi utilisé sur deux amphores Gauloise 5 et apparaît encore quatre fois sur des amphores dont, faute de lèvre, nous ne savons pas si elles appartiennent au type Gauloise 3, 4 ou $5^{277}$. Au total, il qualifie une fois de l'aminneum et huit fois du vin vetus. Massicum est un terme ancien, associé au très célèbre vin du Falerne: on lit, par exemple, Fal(ernum) Mas(sicum) sur une amphore Dressel 1, en 102 av. J.-C. (CIL, XV, 4554). Le Massique était considéré soit comme un clos du Falerne, soit comme un cru indépendant (Tchernia, 1986, p. 342). On en trouve encore la trace entre 37 et 4 av. J.-C., sur une Dressel 2/4 à Massada (Israël) : Mas(sicum) excel(lens) (Cotton, Geiger, 1989, n819).

En Gaule, nous avons l'exemple de deux amphores de forme identique et même standardisée, Gauloise 4 (Laubenheimer, Gisbert Santonja, 2001), contenant toutes les deux de l'aminneum, l'un de Béziers, l'autre du Massique (fig. 89) ! Si l'on a pris la peine de le préciser, c'est qu'il s'agit de qualités différentes. Il est donc important que l'acheteur sache lire et ne se fie pas à la forme de l'amphore, qui sert aussi, on l'a vu, à d'autres sortes de vins gaulois. Le qualificatif vin du Massique fait question. Désigne-t-il une origine ou bien une qualité comme un vin aminné, ou vieux "à la façon campanienne "? C'est sans doute le cas, nous l'avons vu, pour les sauces de poisson "à la façon espagnole » ou « à la façon

275. L'amimnea vitis est un cépage très célèbre dans le monde romain (Columelle, De re rustica, III, 2, 7-13 ; Pline, Histoire naturelle, XIV, 21-22) dont la culture qui comnait plusieurs variétés est encore mentionnée dans l'édit de Dioclétien. Le picatum est un vin poissé lors de la vinification (Columelle, op. cit.. III, 12, 23) ou qui a un goût naturel de poix che\% les Allobroges (Pline, op. cit., XIV, 18). Le mulsum est un vin miellé (Columelle, op. cit. 12, 41), le depletum serait un vin soutire (Liou, Marichal, 1978, p. 155). On trouve également du passum, dans une Gauloise $3 / 5$ (cf. infra catalogue, p. 169, $\mathrm{n}^{\circ} 3$ ), vin fait arec des raisins confits sur pied dont les Voconces font une spécialité (Pline, op. cit., XIV, 8()-84)

276. Linscription complète Massicum a conduit B. Liou à développer l'abréviation courante Mas en Massicum plutôt qu'en Mas(siliense), de Marseille.

277. Sont classées sous le terme Gauloise $3 / 5$ des amphores dont la leve manque et qui pourraient appartenir au type (aduloise 3, ou Gauloise 4, ou encore Gauloise 5 .
d'Antibes " dans des amphores gauloises. S'agit-il au contraire de vin italien, importé en vrac et mis en amphores à l'arrivée en Gaule ${ }^{278}$ ? Dans ce cas, pourquoi ne serait-il pas mis en tonneaux, dont l'usage s'est rapidement développé à cette époque (Marlière, 2002), ou dans un autre contenant que ceux qui caractérisent précisément les vins gaulois?

Un nombre est indiqué parfois à la ligne C. On remarque rapidement plusieurs catégories (tabl. XVII) :

- une contenance, trois modii et cinq sextarii sur une amphore Gauloise 3/5 d'Amiens (n²7), est exprimée comme sur les amphores à poisson Lyon $3 \mathrm{~A}$;

- on reconnaîtra sans doute un poids, pour un nombre dont l'unité n'est pas indiquée mais qui oscille entre 53 et 61 (livres probablement), pour une Gauloise 4 ( $\left.\mathrm{n}^{\circ} 14\right)$, une Gauloise 5 $\left(n^{\circ} 3\right)$ et trois Gauloise 3/5 $\left(n^{\circ s} 18,23,38\right)$. Ceci rappelle bien sûr le poids indiqué de la même façon sur les amphores à poisson Lyon 3B, qui sont un peu plus lourdes ;

- les lettres C, ou CC, ou CCC, ou CCCC, sont utilisées sur huit amphores: Gauloise $4\left(\mathrm{n}^{\circ} 19,20,21,22,23,26\right)$ et Gauloise $3 / 5\left(n^{\text {os }} 34,37\right)$. Il s'agit d'un nombre rond (ni un poids ni un volume) qui est toujours accompagné du qualificatif vetus. S'agirait-il de l'âge du vin, mais quelle serait l'unité employée? Une amphore à poisson Pélichet 46 de Mayence (Ehmig, 2003 : titulus 48) indique l'âge de la saumure, quatre ans : AAAA et en dessous CCC, il s'agit donc d'un qualificatif différent. On peut penser aussi au numéro d'un lot d'amphores évalué par centaines ${ }^{279}$;

- enfin restent inexpliqués des nombres comme D (5 ans ?) sur une Gauloise $4\left(n^{\circ} 13\right)$ ou DCL sur une Gauloise 3/5 (n 45$)$.

À la dernière ligne de l'inscription horizontale, en $\mathrm{D}$, vient le nom du commerçant, parfois tria nomina, parfois simple cognomen. On note, rarement, des erreurs de graphie, un prénom répété deux fois (G. $\left.4, \mathrm{n}^{\circ} 5\right)$ ou une lettre manquante dans un nom (G. 4, n⿳7). Une formule amusante sur une Gauloise $4\left(n^{\circ} 24\right)$ précise que l'amphore est destinée à l'usage personnel du commerçant, suivant l'expression habituelle: usib(us) Iuli Adiutoris, c'est-à-dire « à l'usage de Julius Adiutoris " (Desbat et al., 1987, p. 142). Notons qu'il a choisi le vin qui était probablement le meilleur, de l'aminneum vetus!

En oblique, près de l'anse droite, des inscriptions secondaires indiquent le plus souvent des noms, sans doute liés à des contrôles, ou des précisions difficiles à saisir.

Le style d'écriture utilisé sur les amphores vinaires est variable; les unes sont régulières, les autres chargées de paraphes difficilement lisibles. Elles mériteront une étude complémentaire s'appuyant sur des exemples bien datès.

Comme pour les amphores à poisson, on notera que les mêmes emballages pouvaient contenir des produits différents, par exemple du vin de Béziers dans des Dressel 2/4 ou dans des Gauloise 4, du vin du Massique dans des Gauloise 4 ou dans des

278. Ia livaison en Gaule de vins en vac, transportés dans des dolia à poste fixe de bateaux venus d'Italic ou de Tarraconaise, ne fait pas de doutc (voir en dernier lieu Gianfrotta, 1998).

279. Un svstème équivalent a peut-être étć utilisé sur une amphore Crétoise 4, egalement vinaire ct contemporaine des Gauloise 4, découverte à Fos-sur-Mer, le nombre CC. a été peint sur le col (Liou, 1987, p. (91). 


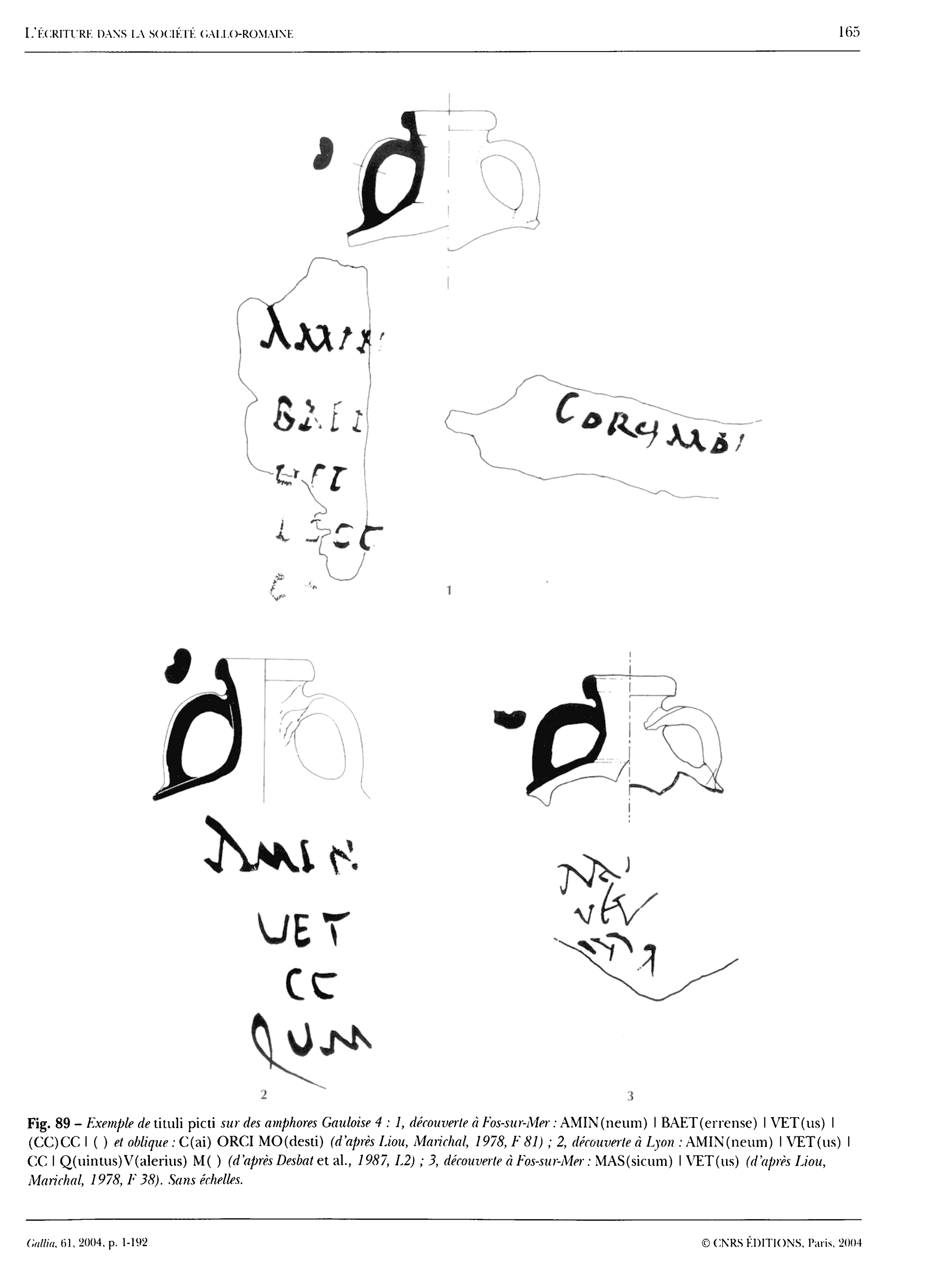


Gauloise 5. La forme de l'amphore ne suffisait pas à informer sur la qualité du contenu, d'où la nécessité de savoir lire les tituli picti pour décoder la nature précise du produit vendu.

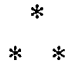

La richesse du corpus des inscriptions peintes sur les amphores gauloises est une nouveauté que l'on commence à explorer. Diversité des récipients, diversité des produits, de leur désignation et de leurs qualificatifs, diversité des écritures, des pratiques de mesure, des habitudes commerciales, de transport, de contrôle, c'est tout un pan de l'histoire de la production, du commerce et de la vie quotidienne en Gaule qui peu à peu s'entrouvre. Pour écrire et donner à lire, les données essentielles à propos des denrées mises en amphores et commercialisées, on s'est inspiré de modèles italiens ou espagnols, mais il apparaît aussi que s'est développé un style "à la gauloise ".

Victor Hugo ne disait-il pas : « car le mot, qu'on le sache, est un être vivant!".

\section{CATALOGUE DES TITULI PICTI SUR AMPHORES GAULOISES}

\section{AMPHORES À POISSON}

\section{DRESSEL 16}

\section{Londres}

horizontal: LIQVAMEN | ANTIPOL(itanum) | EXC(ellens) । L(ucii) TETT(i) AFRI । CANI

(Laubenheimer, 1989 ; Laubenheimer dir., 1992 ; MartinKilcher, 1994, fig. 185 p. 417).

\section{Rome}

horizontal : LIQ(uamen) | ANTI(politanum) | EXC(cellens) | L.V() $M() \mid M I L$

(Dressel : CIL, XV, 4712 ; Martin-Kilcher, 1990, n. 14).

\section{Augst P 55}

horizontal : $M V R($ ia $)|F L(O S)| L($ ou P) $A V R()$

(Martin-Kilcher, 1994, p. 427).

\section{Ostie}

horizontal : MVRI(ae) | FLOS | ANTIPOL(itanum)

(Laubenheimer dir., 1992, fig. 5 p. 19) (Dressel 16 ou

Dressel 2/4 ?).

\section{Narbonne PN 25}

horizontal : M(uriae) F(los) | NTIPOLI(tanae) | N.XV |C(ai) VIBI SECVNDI

(Liou, 1993, p. 144).

\section{Augst}

horizontal : G(arum) ANT(ipolitanum) I FLOS

(Martin-Kilcher, 1990, fig. 3 p. 179).

\section{Bretagne}

horizontal : M(uria) ANIIPOL(itana)

(RIB, 2492, 29).

\section{LYON 3 (COLS INCOMPLETS)}

\section{Koenigshoffen}

vertical : C IVL RNT XVI

(Baudoux, 1996, p. 78, fig. 34, nº).

\section{Vindonissa}

horizontal : (l)I(quamen) I ANT(ipolitanum)

vertical : M.V.P. VIII

(Martin-Kilcher, 1994, fig. 185 p. 417).

\section{Mandeure}

horizontal : $M V R(i a)$
(Jeannin, Laubenheimer, 1989, fig. 13).

\section{Mandeure}

horizontal : $R \cdot V() F()$

(Jeannin, Laubenheimer, 1989, fig. 13).

\section{Vindonissa}

horizontal : $L X I I I \mid M V R$

(Ettlinger, 1977, p. 9 et fig. 2).

\section{LYON 3A}

\section{Lyon L 16, rue des Farges}

horizontal : G(ari) $F($ los) | M(odii) III

vertical : RVSTICI.IV

(Desbat et al., 1987, p. 154).

2. Amiens 2, bibliothèque universitaire

horizontal : G(ari) F(los) | . . | _ - | M(odii) III S(extarii) III

(Marlière, 2000, p. 302, n²).

\section{Koenigshoffen SK 145}

horizontal : (Garum) $S C$ (ombri) | A() | M(odii) I.. ou M(arcus) L.() (Baudoux, 1996, p. 78, fig. 34, $n^{\circ} 9$ ).

\section{Angers, couvent des Jacobins}

horizontal : G(arum) $F($ los) $P() \mid M($ odii) III, S(extarii) I $|M V R()| L()$

(Siraudeau, 1988, pl. 6).

\section{Mayence 37}

horizontal : G(arum) F(los) M(odii) I(- -)

vertical : M.A.F.L । $X X X X$

(Ehmig, 2003, p. 253).

\section{Mayence 38}

horizontal : $G$ (arum) $F($ los $)$ I $M$ (odii) III

vertical : illisible

(Ehmig, 2003, p. 253)

7. Mayence 39

horizontal : G(arum) F(los) | M(odii) III

(Ehmig, 2003, p. 253)

\section{Mayence 40}

horizontal : G(arum) $S C$ (ombri) $\mid M$ (odii) III

vertical : $L$ () $Q($ ) $A P$ ( ) $P($ ) $X X L$

(Ehmig, 2003, p. 254).

U. Ehmig classe cette amphore comme Dressel 9 similis ou Lyon 3B, je pense qu'il s'agit plutôt d'une Dressel 10 similis ou Lyon 3A. 


\section{Mayence 41}

horizontal : $G($ arum) $F($ los $) S C($ ombri) I $M($ odii) $\mathrm{N}$

vertical : $X X \mid C() . I() . G()$.

(Ehmig, 2003, p. 254).

\section{Mayence 42}

horizontal : $G($ arum) $F($ los $) S C$ (ombri) I M(odii) III S(extarii) III vertical : (- - ) T.H.XV

(Ehmig, 2003, p. 254).

11. Mayence 43

horizontal : $M$ (odii) $I I I$

vertical : $C(-$-) $A(--) C(--) X$

(Ehmig, 2003, p. 254).

12. Mayence 44

horizontal : $L X X \mid M$ (odii) III

vertical : $A(--) P(--)$ IIIXV

(Ehmig, 2003, p. 255).

13. Mayence 76

horizontal : (- -) $V(--)$

vertical : (- -) XXI(- - )

(Ehmig, 2003, 76, p. 265).

\section{LYON 3B}

\section{Augst $P 42$}

horizontal : $L X X I \mid M . M() . C L()$

vertical : MASCVIII.V

(Martin-Kilcher, 1994, p. 422).

\section{Augst $P 45$}

horizontal : illisible

vertical : $(S) E X(t i) . I()$

(Martin-Kilcher, 1994, p. 422).

\section{Augst P 52}

horizontal : LXXI ()RSTI

vertical : G.P( ) $C$ ( ) $I I($ )

(Martin-Kilcher, 1994, p. 422).

\section{Augst P 54}

horizontal : $L X())$ ( ) $M()$

(Martin-Kilcher, 1994, p. 422).

\section{Augst $P \mathbf{4 0}$}

horizontal : G(arum) HISP(anum) | LXXI | M.M() CL()

vertical : FRONTINVS $V$

(Martin-Kilcher, 1994, p. 422).

\section{Augsbourg}

horizontal : $\operatorname{GAR}($ um) $\mid \mathrm{EXC}$ (ellens) | T(itus)

(Ehmig, 2000, fig. 9 p. 62).

7. Mayence 36

horizontal : $G A R(u m)|L X I I I I| T() L() M()$

vertical : L. V.F. $X$.

(Ehmig, 2003, p. 253).

\section{Lyon L 11}

horizontal : $M V R(i a)$ । VI

(Desbat et al., 1987, p. 150 ; Desbat, Dangréaux, 1997, p. 88).

9. Strasbourg ST 178

horizontal : $M V R($ ia $) A A()$

(Baudoux, 1996, p. 78, fig. 35, n²).

10. Strasbourg ST 165

horizontal : M(uria) HISP(ana) | EXC(ellens) | - - | XXIII

(Baudoux, 1996, p. 78, fig. 35, nº 6).

\section{Augst P 41}

horizontal : MVR(ia) HISP(ana) I LXXII $\mid M$. CL()

vertical : MASCVII. $V$

(Martin-Kilcher, 1994, p. 422).

\section{Augst $P 43$}

horizontal : $\operatorname{MVR}(i a) \mid(M) M()$. CI. ()

vertical : $M . T($ ). $M($ )

(Martin-Kilcher, 1994, p. 422).

\section{Augst $P 44$}

horizontal : $M V R(i a)|(H) I S P(a n a)| I X()$

vertical : $\operatorname{SEX}(t i) . I V$.

(Martin-Kilcher, 1994, p. 422).

\section{Augst $P 46$}

horizontal : MVR(ia) | - - I EXC(ellens) | LXX |M. M( ) CL()

vertical : $C . P() . F()$ IIII

(Martin-Kilcher, 1994, p. 422).

\section{Augst $P 47$}

horizontal : MVR(ia) | ANTI(politana) | EXC(ellens) | (L)XX

vertical : C. $P() . C()$. IIII

(Martin-Kilcher, 1994, p. 427).

\section{Augst $P 48$}

horizontal : $M V R(i a)$ I (L)XX

vertical : Q.S( ). $L()()$

(Martin-Kilcher, 1994, p. 422).

\section{Augst $P 49$}

horizontal : MVR(ia) I ()XTANI?

vertical : ().R.S II()

(Martin-Kilcher, 1994, p. 422).

\section{Augst P 50}

horizontal : MVR(ia) I HIS(pana)?

(Martin-Kilcher, 1994, p. 422).

\section{Augst P 51}

horizontal : $M V R($ ia $)|L X X|() L()$

vertical : $M . E() R($ ) IIII ou M.F() $R($ ) IIII

(Martin-Kilcher, 1994, p. 422).

\section{Augst P 53}

horizontal : $M V R(i a) \mid L()$

(Martin-Kilcher, 1994, p. 422).

21. Mayence, titulus 46

horizontal : $M V R(i a)$

(Ehmig, 2003, p. 255).

22. Mayence, titulus 77

vertical : $L E G$ (ionis) ?

(Ehmig, 2003, p. 265).

\section{LYON 4}

\section{Augst 84}

horizontal : $G$ (ari) F(los) | Excellens

(Martin-Kilcher, 1994, p. 381, fig. 156, n²).

\section{Vindonissa}

horizontal : G(ari) F(los) | _ - । XXI

(Martin-Kilcher, 1994, p. 381, fig. 156).

3. Lux, musée de Chalon-sur-Saône inv. 87 20-2

horizontal : illisible | IIII(-) | illisible

vertical : $S(-) L(-) S I$ 


\section{Augst 34}

\section{Vindomissa}

horizontal : MVR(iae) F(los) I M IIII S VIII.

(Martin-Kilcher, 1994, fig. 184 p. 416).

\section{AMPHORETTE}

1. Lyon $\mathrm{L} 17$

horizontal : LIQ(uamen) | APIC() | G.RVFI

(Desbat et al., 1987, p. 154).

\section{AMPHORES À OLIVES}

\section{AUGST 21}

\section{Londres, Walbrook}

horizontal : (O)LIV(ae)

vertical : inscription non déchiffrée.

(Sealey, Tyers, 1989, p. 63 et pl. VIIIb).

\section{Colchester}

horizontal : OLI(vae) ? I CLP

(Sealey, Tyers, 1989, p. 63 et fig. 4).

3. Vechten V.F. 197

horizontal : OL(ivae) I A(lbae) ?

(Martin-Kilcher, 1994, $\mathrm{n}^{\circ} 472$ p. 392).

\section{Londres, Cornhill}

horizontal: OL(ivae) | AL(bae) | CCL | CIA | VIANV ou AMV (Sealey, Tyers, 1989, p. 63).

\section{Augst P 24}

horizontal: (O)LI(vae) | EX $\mid I V L(c i)$

(Martin-Kilcher, 1994, fig. 169 p. 392).

6. Nyon

horizontal: OLI(vae) । (albae ou nigrae) । (ex) DVL(ci) ।

EXC(ellens) I M. CIR()

(Pélichet, 1946, fig. 11 p. 198).

\section{Vindolanda}

horizontal : OL(ivae) $A L($ bae $)$

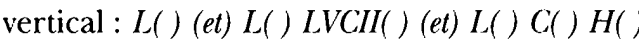

(Marlière, 2003, fig. 19 p. 158).

8. Mayence, titulus 9

horizontal : OL(ivae) | AL(bae)

(Ehmig, 2003, p. 248).

\section{AMPHORES À VIN}

DRESSEL 2/4

\section{Rome, Castro Pretorio}

horizontal : $S V M|V E T(u s)| V \mid$ BAETERR(ense) |

L. MARTI SATVLLI

vertical : $F V L()$

(CIL, XV, 4542 : Dressel, 1879, p. 63).

2. Rome, Castro Pretorio

horizontal : BAETER(ense) | ALB(um)

(CIL, XV, 4543).

\section{Gauloise 7}

1. Fréjus

horizontal : L(ucio) (iunio) SIIANO C(aio) V(ellaeo) TVTORE $C O(n) S$ (ulibus) | AMINN(eum) T( , LOC()

(Liou, 1991).

\section{Ostie}

horizontal : $\operatorname{CIRT}(a)$ ou (IRT(ensis)

(Carta et al., 1978).

\section{GAULOISE 3}

\section{Fos-sur-Mer F 104}

horizontal : $A M I N N($ eum $)$

(Liou, 1987, p. 72).

2. Fos-sur-Mer F 107

horizontal : V(inum) AM(inneum) ?

(Liou, 1987, p. 74).

\section{GAULOISE 4}

\section{Fos-sur-Mer F 38}

horizontal : MAS(sicum) | VETV(s)

(Liou, Marichal, 1978, n 38 p. 147).

2. Fos-sur-Mer $\mathbf{F} 48$

oblique : $A$. CVII

(Liou, Marichal, 1978, n 48 p. 153).

3. Fos-sur-Mer F 52

horizontal : $V($ inum) ? DEP(letum) ?

(Liou, Marichal, 1978, n52 p. 155).

4. Fos-sur-Mer $\mathbf{F} 53$

horizontal : TEN ?

(Liou, Marichal, 1978, n53 p. 157).

5. Fos-sur-Mer F 109

horizontal: MAS(sicum) | VET(us) । Q.QVINTI HELVI । MARTIALIS

(Liou, 1987, no 109 p. 76).

6. Fos-sur-Mer F 110

horizontal : MAS(sicum) | VET(us) I T(itus) MARI()

(Liou, 1987, p. 76).

7. Fos-sur-Mer F 115

horizontal : EN ? I L(uci) ANNAEI EPAP(h)RODITI

(Liou, 1987, p. 82).

8. Fos-sur-Mer F 120

horizontal : PHILETI

(Liou, 1987, p. 85).

9. Marseille M 3, la Bourse

horizontal : bac | mu(lsum) ? I ad | viatoris

(Liou, 1987, p. 104).

10. Marseille M 4, la Bourse

horizontal : $C() \mid C($ ?) $M O N|| A R$

(Liou, 1987, p. 104).

11. Calvi

oblique : ARQVAN()

(Liou, 1987, p. 136)

12. Lyon $L$ 4, Bas de Loyasse

horizontal : MAS(sicum) I VET(us)

(Desbat et al., 1987, p. 144).

13. Lyon L 6, ancien couvent des Capucins horizontal : VET(us) $|\operatorname{IEC}()| I) \mid P I()$

(Desbat et al., 1987, p. 146).

14. Lyon $L 7$, Bas de Loyasse horizontal : LV

oblique : ris(?)com(?) domes(tici)

(Desbat et al., 1987, p. 148). 


\section{Sud de l'Angleterre}

horizontal : MAS(sicum) | VET(us)

(inćdit, comm. D. Williams).

16. Augst, inv. 2974

horizontal : VET(us)

(Martin-Kilcher, 1994, p. 366).

\section{Augsbourg}

horizontal : $M() G()()$

(Ehmig, 2000, fig. 18 p. 66).

18. Mayence, titulus 30

horizontal : $A M I N($ neum $)$

oblique : CARCI

(Ehmig, 2003, p. 252).

19. Fos-sur-Mer F 81

horizontal : AMIN(neum) I BAET(errense) I VET(us) I ??CC: I ? (Liou, Marichal, 1978, p. 179).

20. Lyon L 2, Bas de Loyasse

horizontal : $A M I N($ neum) | VET(us) | CC। $Q$ () $V() M()$

(Desbat et al., 1987, p. 144).

21. Mayence, titulus 27

horizontal : $A M I N($ neum) | VET(us) | CCC:T( ) T( ) N()

oblique : $H Y C A N I$

(Ehmig, 2003, p. 251).

22. Mayence, titulus 28

horizontal : $A M($ inneum) | $V E T(u s)|C C C:| T() E() C()$

oblique : $(:$

(Ehmig, 2003, p. 25l).

23. Mayence, titulus 29

horizontal : AMIN(eum) | VET(us) | C () NICI

oblique : BVBALI

(Ehmig, 2003, p. 251).

24. Lyon $\mathrm{L} 1$, dépotoir de Trion

horizontal : AMIN(neum) | VEIT(us) VSIB(us) | IVLI ADIVTORI

(Desbat et al., 1987, p. 142).

25. Fréjus, plate-forme

horizontal : PIC(atum) | V(etus) | $M() A() P()$

oblique : IVGOLI

(Liou, 1992, fig. 9 p. 93).

26. Augst, inv. 865788

horizontal : PIC(atum) | VET(us) | III

(Martin-Kilcher, 1994, fig. 15̆l p. 376).

27. Mayence, titulus 31

horizontal : $P I($ (atum) । VET(us)

(Ehmig, 2003, p. 252).

28. Mayence, titulus 32

horizontal : illisible

oblique : illisible

(Ehmig, 2003, p. 252).

29. Mayence, titulus 33

oblique : TVIIIII T( )BI

(Ehmig, 2003, p. 252).

30. Mayence, titulus 34

oblique : P IIII

(Ehmig, 2003, p. 252).

31. Mayence, titulus 35

horizontal : $L O O C$

(Ehmig, 2003, p. 252).
32. Yverdon-les-Bains

horizontal : MAS(sicum) | VET(us) I CCC:।()

oblique : CAA I CACXXIII

(Martin-Kilcher, à paraître).

33. Avenches

horizontal : P.MARI(us) $C O(n) S(u l)$

oblique : $C()$

(Martin-Kilcher, à paraître).

\section{GaUloise 5}

\section{Fos-sur-Mer F 116}

oblique : Ati(li)

(Liou, 1987, p. 82).

2. Fos-sur-Mer F 117

oblique : ebirense ccxii

(Liou, 1987, p. 82).

3. Fos-sur-Mer F 39

horizontal : MAS(sicum) |LXI (sextarii) | OBEILIOIO(rum)

oblique : MILESI

(Liou, Marichal, 1978, p. 147, n³9).

4. Newstead (Écosse), musée d'Édimbourg inv. FRA 1433 horizontal : ATTI SECVNDI

(Laubenheimer, 1985, tabl. 29).

5. Fos-sur-Mer F 114

horizontal : $P I C($ alum $) \mid$ EXC(ellens) I C.A.C

(Liou, 1987, p. 82).

6. Freidberg-les-Augsburg

horizontal : MAS(sicum) | VET(us) I CC(C) ( )R

vertical : $I I I()$

(Sorge, 1999).

\section{GAULOISE 3/5 (FRAGMENTS SANS LÈVRE)}

\section{Fos-sur-Mer $F 37$}

horizontal : $A M(i) N($ neum $)$

(Liou, Marichal, 1978, n”37 p. 147).

2. Fos-sur-Mer F 40

horizontal : VET(us) I MAS(siliense) | ???OBELLIOR(um)

(Liou, Marichal, 1978, n40 p. 149).

3. Fos-sur-Mer F 41

horizontal : PASS(um)

(Liou, Marichal, 1978, n 41 p. 149).

4. Fos-sur-Mer F 47

oblique : CATVRI

(Liou, Marichal, 1978, n” 47 p. 153).

5. Fos-sur-Mer F 49

oblique : NEONIS । MACI

(Liou, Marichal, 1978, p. 153).

6. Fos-sur-Mer F 50

horizontal : POLYTIMI

(Liou, Marichal, 1978, p. 155).

7. Fos-sur-Mer F 51

horizontal : $C I() \mid$ ( )TI। $M()$

(Liou, Marichal, 1978: p. 155).

8. Fos-sur-Mer F 101

horizontal: IMP(eratore) VESP(asiano) AVG(usto) $V$ (?)

T(ito imperatore III ? consulibus)

(Liou, 1987, p. 70). 


\section{Fos-sur-Mer F 102}

horizontal : (Imperatore) (Vesp)ASIANO VIIII I

(Tito) (I)MP(eratore) VII (consulibus)

(Liou, 1987, p. 72).

Le neuvième consulat de Vespasien et le septième

de Titus correspondent à l'année 79.

10. Fos-sur-Mer F 108

horizontal : MASSICVM । CM?

(Liou, 1987, p. 74).

11. Fos-sur-Mer F 118

horizontal : $E L$

(Liou, 1987, p. 85).

12. Fos-sur-Mer F 119

horizontal : $L$ CASSI

(Liou, 1987, p. 85).

13. Fos-sur-Mer F 121

horizontal : PRIMIGENIO | DECVMI

(Liou, 1987, p. 85).

14. Fos-sur-Mer F 122

horizontal : P.VOLCACI

(Liou, 1987, p. 87).

15. Fos-sur-Mer F 123

oblique : TIMOTHEI

(Liou, 1987, p. 87).

16. Fos-sur-Mer F 124

horizontal : C?.V.C. I CAP ou $R$

(Liou, 1987, p. 87).

17. Fos-sur-Mer F 125

horizontal : () Cordi Callisti.

(Liou, 1987, p. 87).

\section{Fos-sur-Mer F 126}

horizontal : LIII (soit 53 sextarii $=29$ litres).

(Liou, 1987, p. 87).

19. Fos-sur-Mer F 127

oblique : clui (soit le chiffre 156).

(Liou, 1987, p. 87).

20. Marseille M 5

oblique : ()MA() । ()NO() । ()IT()TI। LICINI P(?)F()

(Liou, 1987, p. 104).

21. Lyon $L$, ancien couvent des Capucins

oblique : vinum lutat 1 proxsum

(Desbat et al., 1987, p. 146).

22. Lyon L 8, Bas de Loyasse

oblique : pri...ll(i)

(Desbat et al., 1987, p. 148)

23. Lyon $L$ 9, Bas de Loyasse

horizontal : $L X$

(Desbat et al., 1987, p. 148).

24. Lyon L 10, ancien couvent des Capucins

horizontal : grands paraphes illisibles.

(Desbat et al., 1987, p. 148).

25. Rome 221a, Testaccio

oblique : TRYPHON(is)

Écriture à grands paraphes, datée de 150-180.

(Blásquez Martínez dir., 1994, p. 128).

26. Rome 222, Testaccio

oblique : ()ses

(Blásquez Martínez dir., 1994, p. 128).
27. Amiens 2, Bibliothèque universitaire 1995

horizontal : IIIS $V$ (soit 3 modii et 5 sextarii $=29,5$ litres).

(Marlière, 2000, p. 289, $\mathrm{n}^{\circ}$ 2).

28. Amiens 3, Palais des Sports 1993

horizontal : illisible

(Marlière, 2000, p. 298, $\mathrm{n}^{\circ} 3$ ).

29. Fos-sur-Mer F 36

horizontal : AMINNEVM

(Liou, Marichal, 1978, p. 145, $\mathrm{n}^{\circ} 36$ ).

30. Fos-sur-Mer F 103

horizontal : AMINNEVM

(Liou, 1987, p. 72).

31. Fos-sur-Mer F 105

horizontal : $A M I N($ neum $)$

(Liou, 1987, p. 72).

32. Fos-sur-Mer F 106

horizontal : $A M I N($ neum) I VET(us)

(Liou, 1987, p. 72).

33. Fos-sur-Mer F 111

horizontal: MAS(sicum) । AMIN(neum) | T(itus) AELI(us) HERRIANI(us)

oblique : EARINI

(Liou, 1987, p. 76).

34. Lyon $L$ 3, rue des Farges

horizontal : $A M I N($ neum $) \mid \operatorname{VET}($ us $)|C C C| T() E() N()$

(Desbat et al., 1987, p. 144).

35. Rome $\mathbf{n}^{\circ}$ 221d, Testaccio

horizontal : $A M I N($ neum $) \mid V E T($ us $)|M Y()| F E I B()$

oblique : PHILANT( )

(Rodríguez-Almeida, 1984, fig. 44 p. 115 ; Blásquez Martínez dir., 1994, p. 127).

36. Amiens 1, Palais des Sports 1992 (PDS 92)

horizontal : $\operatorname{AMIN}($ neum) । $\operatorname{VET}($ us)

Écriture à arabesques, datée de 140-200.

(Marlière, 2000, p. 297, n 1).

37. Mandeure, fouille 1999

horizontal : MAS(sicum) | VET(us) | CCCC | M( ) I( )

(Mazimann, 2002).

38. Fos-sur-Mer F 112

horizontal : $M V L S($ um) AR(omatica) $|L X|$ Q. I. T. | POM . .

Le chiffre LX (soit 60 sextarii $=32,8$ litres).

(Liou, 1987, p. 78).

39. Fos-sur-Mer F 113

horizontal : $M V($ lsum) । $C B$ ?

(Liou, 1987, p. 78).

40. Gaujac

horizontal : $M V(l$ sum $) \mid N$ ?

(Laubenheimer, 1985, p. 448).

41. Fos-sur-Mer F 42

horizontal : PICAT(um) | VET(us)

(Liou, Marichal, 1978, no 42 p. 149).

42. Fos-sur-Mer F 43

horizontal : PIC(atum) । VET(us)

oblique : IITVLI

(Liou, Marichal, 1978, n 43 p. 151).

43. Fos-sur-Mer F 44

horizontal : $P I C($ atum)

oblique : $S T Y R()$ 
(Liou, Marichal, 1978, n44 p. 151).

\section{Fos-sur-Mer F 45}

horizontale : PICA(tum)

(Liou, Marichal, $1978, n^{\circ} 45$ p. 153).

\section{Toulon $\mathrm{T} 2$}

horizontal : PIC(atum) | VET(us) | DCL | POITIOR(um)

Le nombre DCL (650) n'est pas une contenance.

(Liou, 1987, p. 110).

46. Épave Saint-Gervais 3

horizontal : PIC(atum)

(Liou, Gassend, 1990, p. 212).

47. Toulon $\mathrm{T}^{\text {bis }}$

horizontal : PIC(atum)
(Liou, Gassend, 1990, n. 96).

\section{Mataro}

horizontal : AMIN(eum) | VET(us) | M()

(Revilla, 2000-2001).

Il pourrait aussi s'agir d'une Gauloise 4 fabriquée localement. 49. Braughing

horizontal : ? ILV()

(Fitzpatrick, 2003, fig. 11 p. 21).

50. Vindolanda

horizontal : $\operatorname{VNE}($ ) ? I VE(tus)

oblique : $C I I$ ?

(Marlière, 2003).

La lecture n'est pas satisfaisante. 